UMN-TH-2617/07

FTPI-MINN-07/27

September 2007

\title{
Colliders and Cosmology
}

\author{
Keith A. Olive ${ }^{1}$ a \\ William I. Fine Theoretical Physics Institute, University of Minnesota, Minneapolis, MN 55455, USA
}

\begin{abstract}
Dark matter in variations of constrained minimal supersymmetric standard models will be discussed. Particular attention will be given to the comparison between accelerator and direct detection constraints.
\end{abstract}

PACS. XX.XX.XX No PACS code given

\section{Introduction}

Evidence for dark matter in the universe is available from a wide range of observational data. In addition to the classic evidence from galactic rotation curves [1] the analysis of the cosmic microwave background anisotropies leads to the conclusion that the curvature of the universe is close to zero indicating that the sum of the fractions of critical density, $\Omega$, in matter and a cosmological constant (or dark energy) is close to one [2]. When combined with a variety of data including results from the analysis of type Ia supernovae observations [3] and baryon acoustic oscillations [4] one is led to the concordance model where $\Omega_{m} \sim 0.23$ and $\Omega_{\Lambda} \sim 0.73$ with the remainder (leading to $\Omega_{t o t}=1$ ) in baryonic matter. Other dramatic pieces of evidence can be found in combinations of X-ray observations and weak lensing showing the superposition of dark matter (from lensing) and ordinary matter from X-ray gas [5] and from the separation of baryonic and dark matter after the collision of two galaxies as seen in the Bullet cluster [6. For a more complete discussion see [7.

Here, I will adopt the results of the three-year data from WMAP 2] which has determined many cosmological parameters to unprecedented precision. Of particular interest is the determination of the total matter density (relative to the critical density), $\Omega_{m} h^{2}$ and the baryonic density, $\Omega_{b} h^{2}$. In the context of the $\Lambda \mathrm{CDM}$ model, the WMAP only results indicate

$$
\Omega_{m} h^{2}=0.1265_{-0.0080}^{+0.0081} \quad \Omega_{b} h^{2}=0.0223 \pm 0.0007
$$

The difference corresponds to the requisite dark matter density

$$
\Omega_{C D M} h^{2}=0.1042_{-0.0080}^{+0.0081}
$$

or a $2 \sigma$ range of $0.0882-0.1204$ for $\Omega_{C D M} h^{2}$.

\footnotetext{
a Email: olive@umn.edu
}

\section{Constrained MSSM models}

In its generality, the minimal supersymmetric standard model (MSSM) has over 100 undetermined parameters. But in addition to relieving the helplessness of an analysis with so many free parameters, there are good arguments based on grand unification [8] and supergravity [9] which lead to a strong reduction in the number of parameters. I will assume several unification conditions placed on the supersymmetric parameters. In all models considered, the gaugino masses are assumed to be unified at the GUT scale with value, $m_{1 / 2}$, as are the trilinear couplings with value $A_{0}$. Also common to all models considered here is the unification of all soft scalar masses set equal to $m_{0}$ at the GUT scale. With this set of boundary conditions at the GUT scale, we can use the the radiative electroweak symmetry breaking conditions by specifying the ratio of the two Higgs vacuum expectation values, $\tan \beta$, and the mass, $M_{Z}$, to predict the values of the Higgs mixing mass parameter, $\mu$ and the bilinear coupling, $B$. The sign of $\mu$ remains free. This class of models is often referred to as the constrained MSSM (CMSSM) 10, 11, 12,13,14.

Although the CMSSM is often confused with in name with mSUGRA, i.e. models based on minimal supergravity, [15, the latter employ two additional constraints [16]. In addition to the conditions listed above, these are: a relation between the bilinear and trilinear couplings $B_{0}=A_{0}-m_{0}$, and the relation between the gravitino mass and soft scalar masses, $m_{3 / 2}=m_{0}$. When electroweak symmetry breaking boundary conditions are applied, this theory contains only $m_{1 / 2}, m_{0}$, and $A_{0}$ in addition to the sign of $\mu$, as free parameters. The magnitude of $\mu$ as well as $\tan \beta$ are predicted.

I will also discuss a less constrained model, the NUHM, in which the Higgs soft masses are not unified at the GUT scale 17, 18. In this class of models, both $\mu$ and the Higgs pseudo scalar mass become free parameters.

In all of the models discussed below, I will assume unbroken $R$-parity and and hence the lightest super- 
symmetric particle is stable. This will often, but not always be the neutralino [19.

\section{The CMSSM after WMAP}

For a given value of $\tan \beta, A_{0}$, and $\operatorname{sgn}(\mu)$, the resulting regions of acceptable relic density and which satisfy the phenomenological constraints can be displayed on the $m_{1 / 2}-m_{0}$ plane. In Fig. 17a, the light shaded region corresponds to that portion of the CMSSM plane with $\tan \beta=10, A_{0}=0$, and $\mu>0$ such that the computed relic density yields the WMAP value given in eq. (2) [13. The bulk region at relatively low values of $m_{1 / 2}$ and $m_{0}$, tapers off as $m_{1 / 2}$ is increased. At higher values of $m_{0}$, annihilation cross sections are too small to maintain an acceptable relic density and $\Omega_{\chi} h^{2}$ is too large. Although sfermion masses are also enhanced at large $m_{1 / 2}$ (due to RGE running), co-annihilation processes between the LSP and the next lightest sparticle (in this case the $\tilde{\tau}$ ) enhance the annihilation cross section and reduce the relic density. This occurs when the LSP and NLSP are nearly degenerate in mass. The dark shaded region has $m_{\tilde{\tau}}<m_{\chi}$ and is excluded. The effect of coannihilations is to create an allowed band about $25-50 \mathrm{GeV}$ wide in $m_{0}$ for $m_{1 / 2} \lesssim 950 \mathrm{GeV}$, or $m_{1 / 2} \lesssim 400 \mathrm{GeV}$, which tracks above the $m_{\tilde{\tau}_{1}}=m_{\chi}$ contour 20].

Also shown in Fig. [1] are the relevant phenomenological constraints. These include the LEP limits on the chargino mass: $m_{\chi^{ \pm}}>104 \mathrm{GeV}$ [21], on the selectron mass: $m_{\tilde{e}}>99 \mathrm{GeV}$ [22] and on the Higgs mass: $m_{h}>114 \mathrm{GeV}\left[23\right.$. The former two constrain $m_{1 / 2}$ and $m_{0}$ directly via the sparticle masses, and the latter indirectly via the sensitivity of radiative corrections to the Higgs mass to the sparticle masses, principally $m_{\tilde{t}, \tilde{b}}$. FeynHiggs 24 is used for the calculation of $m_{h}$. The Higgs limit imposes important constraints principally on $m_{1 / 2}$ particularly at low $\tan \beta$. Another constraint is the requirement that the branching ratio for $b \rightarrow s \gamma$ is consistent with the experimental measurements 25]. These measurements agree with the Standard Model, and therefore provide bounds on MSSM particles [26], such as the chargino and charged Higgs masses, in particular. Typically, the $b \rightarrow s \gamma$ constraint is more important for $\mu<0$, but it is also relevant for $\mu>0$, particularly when $\tan \beta$ is large. The constraint imposed by measurements of $b \rightarrow s \gamma$ also excludes small values of $m_{1 / 2}$. Finally, there are regions of the $\left(m_{1 / 2}, m_{0}\right)$ plane that are favoured by the BNL measurement 27] of $g_{\mu}-2$ at the 2- $\sigma$ level, corresponding to a deviation from the Standard Model calculation [28] using $e^{+} e^{-}$data.

Another mechanism for extending the allowed regions in the CMSSM to large $m_{\chi}$ is rapid annihilation via a direct-channel pole when $m_{\chi} \sim \frac{1}{2} m_{A}$ [10,12. Since the heavy scalar and pseudoscalar Higgs masses decrease as $\tan \beta$ increases, eventually $2 m_{\chi} \simeq m_{A}$ yielding a 'funnel' extending to large $m_{1 / 2}$ and $m_{0}$ at large $\tan \beta$, as seen in Fig. 10. As one can see, the impact of the Higgs mass constraint is reduced (relative
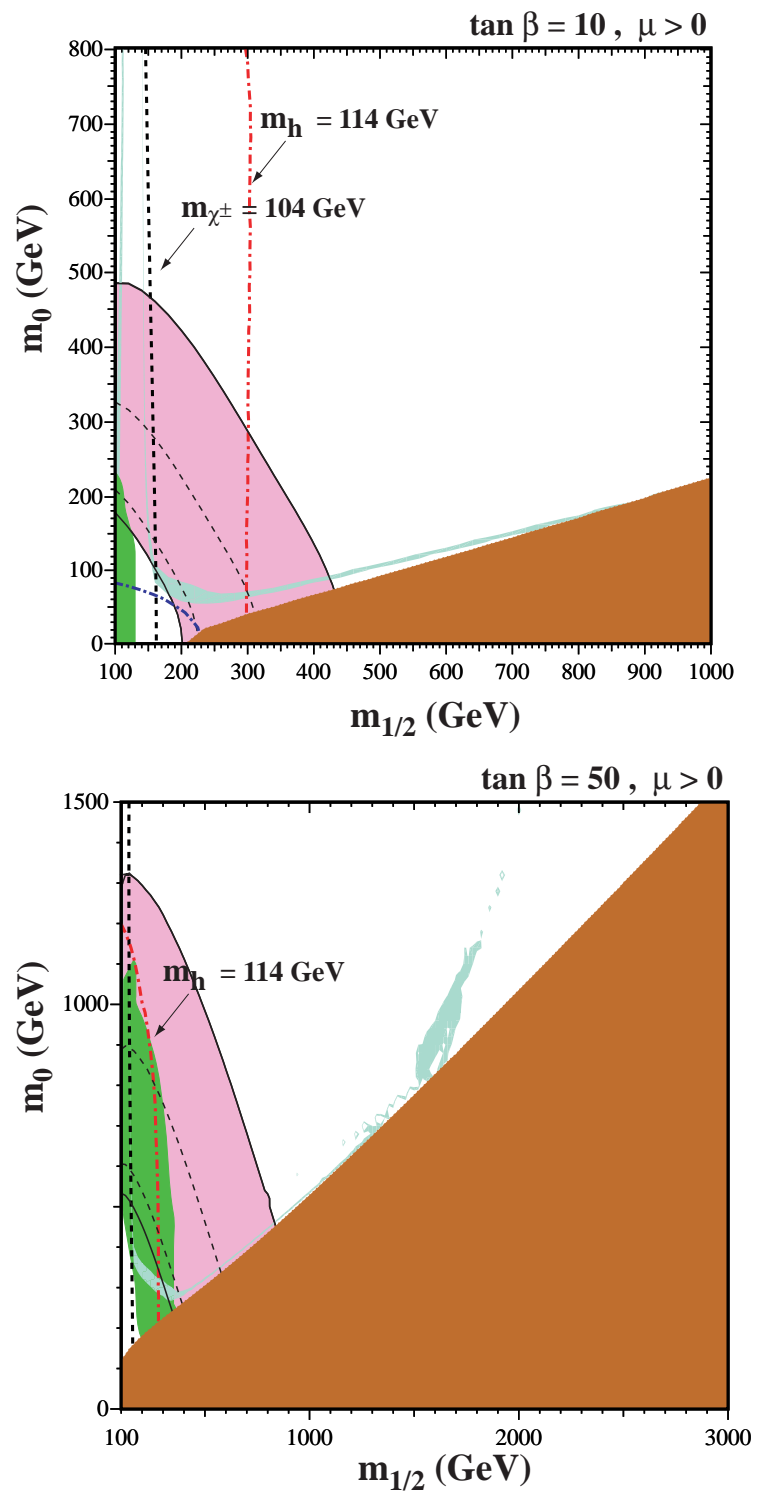

Fig. 1. The $\left(m_{1 / 2}, m_{0}\right)$ planes for (a) $\tan \beta=10$ and $\mu>0$, assuming $A_{0}=0, m_{t}=175 \mathrm{GeV}$ and $m_{b}\left(m_{b}\right)_{S M}^{\overline{M S}}=$ $4.25 \mathrm{GeV}$. The near-vertical (red) dot-dashed lines are the contours $m_{h}=114 \mathrm{GeV}$, and the near-vertical (black) dashed line is the contour $m_{\chi^{ \pm}}=104 \mathrm{GeV}$. Also shown by the dot-dashed curve in the lower left is the corner excluded by the LEP bound of $m_{\tilde{e}}>99 \mathrm{GeV}$. The medium (dark green) shaded region is excluded by $b \rightarrow s \gamma$, and the light (turquoise) shaded area is the cosmologically preferred region. In the dark (brick red) shaded region, the LSP is the charged $\tilde{\tau}_{1}$. The region allowed by the E821 measurement of $a_{\mu}$ at the 2- $\sigma$ level, is shaded (pink) and bounded by solid black lines, with dashed lines indicating the 1- $\sigma$ ranges. In (b), $\tan \beta=50$.

to the case with $\tan \beta=10$ ) while that of $b \rightarrow s \gamma$ is enhanced.

Shown in Fig. 2 are the WMAP lines [13] of the $\left(m_{1 / 2}, m_{0}\right)$ plane for $\mu>0$ and values of $\tan \beta$ from 5 to 55 , in steps $\Delta(\tan \beta)=5$. We notice immediately that the strips are considerably narrower than the spacing between them, though any intermediate 


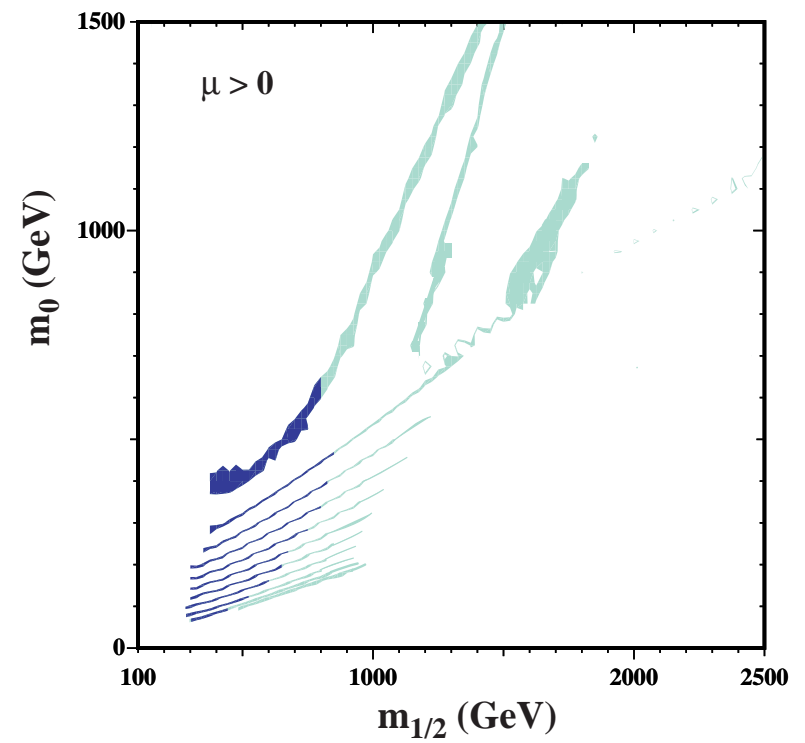

Fig. 2. The strips display the regions of the $\left(m_{1 / 2}, m_{0}\right)$ plane that are compatible with the WMAP determination of $\Omega_{\chi} h^{2}$ and the laboratory constraints for $\mu>0$ and $\tan \beta=$ $5,10,15,20,25,30,35,40,45,50,55$. The parts of the strips compatible with $g_{\mu}-2$ at the $2-\sigma$ level have darker shading.

point in the $\left(m_{1 / 2}, m_{0}\right)$ plane would be compatible with some intermediate value of $\tan \beta$. The right (left) ends of the strips correspond to the maximal (minimal) allowed values of $m_{1 / 2}$ and hence $m_{\chi}$. The lower bounds on $m_{1 / 2}$ are due to the Higgs mass constraint for $\tan \beta \leq 23$, but are determined by the $b \rightarrow s \gamma$ constraint for higher values of $\tan \beta$.

Finally, there is one additional region of acceptable relic density known as the focus-point region [29, which is found at very high values of $m_{0}$. An example showing this region is found in Fig. 3 plotted for $\tan \beta=10, \mu>0$, and $m_{t}=175 \mathrm{TeV}$. As $m_{0}$ is increased, the solution for $\mu$ at low energies as determined by the electroweak symmetry breaking conditions eventually begins to drop. When $\mu \lesssim m_{1 / 2}$, the composition of the LSP gains a strong Higgsino component and as such the relic density begins to drop precipitously. As $m_{0}$ is increased further, there are no longer any solutions for $\mu$. This occurs in the shaded region in the upper left corner of Fig. 3.

\section{Direct detection}

Direct detection techniques rely on the neutralino nucleon scattering cross-section. In general, there are two contributions to the low-energy effective four-fermion Lagrangian which are not velocity dependent. These can be expressed as spin-dependent and scalar or spinindependent interactions. Here I will discuss only spinindependent elastic $\chi$-nucleon scattering stemming from

$$
\mathcal{L}=\alpha_{3 i} \bar{\chi} \chi \overline{q_{i}} q_{i}
$$

which is to be summed over the quark flavours $q$, and the subscript $i$ labels up-type quarks $(i=1)$ and down-

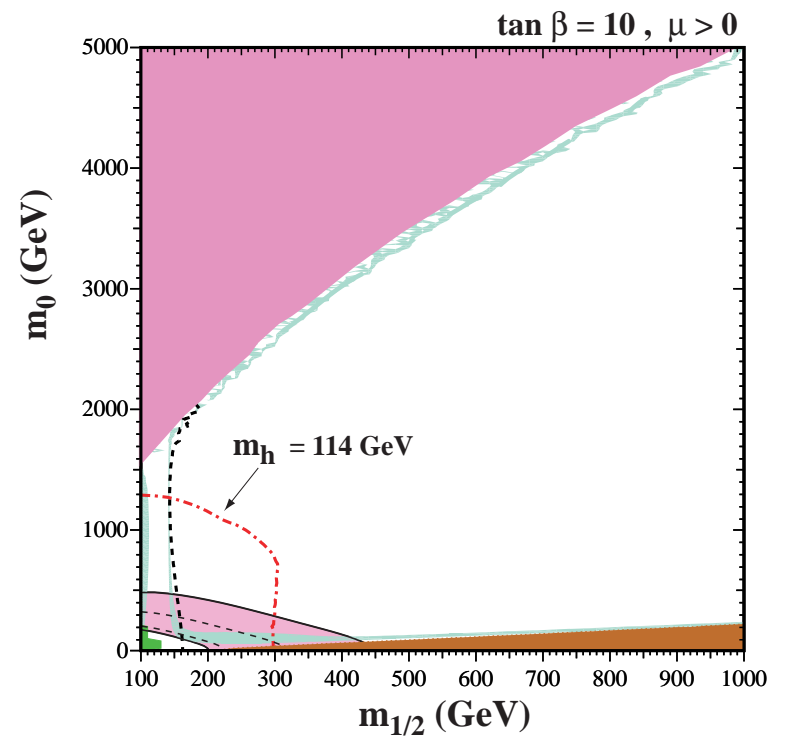

Fig. 3. As in Fig. 1 a, where the range in $m_{0}$ is extended to $5 \mathrm{TeV}$. In the shaded region at very high $m_{0}$, there are no solutions for $\mu$ which respect the low energy electroweak symmetry breaking conditions.

type quarks $(i=2)$. The expression for $\alpha_{3 i}$ can be found in 30 .

The scalar cross section can be written in terms of $\alpha_{3 i}$

$$
\sigma_{3}=\frac{4 m_{r}^{2}}{\pi}\left[Z f_{p}+(A-Z) f_{n}\right]^{2},
$$

where $m_{r}$ is the reduced LSP mass,

$$
\frac{f_{p}}{m_{p}}=\sum_{q=u, d, s} f_{T q}^{(p)} \frac{\alpha_{3 q}}{m_{q}}+\frac{2}{27} f_{T G}^{(p)} \sum_{c, b, t} \frac{\alpha_{3 q}}{m_{q}},
$$

the parameters $f_{T q}^{(p)}$ are defined by

$$
m_{p} f_{T q}^{(p)} \equiv\left\langle p\left|m_{q} \bar{q} q\right| p\right\rangle \equiv m_{q} B_{q},
$$

$f_{T G}^{(p)}=1-\sum_{q=u, d, s} f_{T q}^{(p)}\left[31\right.$, and $f_{n}$ has a similar expression. The needed matrix elements are determined in part by the $\pi$-nucleon $\Sigma$ term, which is given by

$$
\sigma_{\pi N} \equiv \Sigma=\frac{1}{2}\left(m_{u}+m_{d}\right)\left(B_{u}+B_{d}\right) .
$$

and the strangeness contribution to the proton mass,

$$
y=\frac{2 B_{s}}{B_{u}+B_{d}}=1-\frac{\sigma_{0}}{\Sigma}
$$

where $\sigma_{0}$ is the change in the nucleon mass due to the non-zero $u, d$ quark masses, which is estimated on the basis of octet baryon mass differences to be $\sigma_{0}=36 \pm 7$ $\mathrm{MeV}$ 32. Values of the matrix elements are given in the table for 3 choices of $\Sigma$ and hence $y$. As one can see, we expect there will be a strong dependence of the cross section on the proton strangeness, $y$. 
Table 1. Matrix elements in terms of proton strangeness

\begin{tabular}{lllll}
\hline$\Sigma(\mathrm{MeV})$ & $y$ & $f_{T_{u}}$ & $f_{T_{d}}$ & $f_{T_{s}}$ \\
\hline 45 & 0.2 & 0.020 & 0.026 & 0.117 \\
64 & 0.44 & 0.027 & 0.039 & 0.363 \\
36 & 0 & 0.016 & 0.020 & 0.0 \\
\hline
\end{tabular}

\section{Indirect sensitivities}

Measurements of electroweak precision observables (EWPO) as well as B-physics observables (BPO) can provide interesting indirect information about the supersymmetric parameter space. We have already seen the impact of measurements of the anomolous magnetic moment of the muon, the branching raio of $b \rightarrow$ $s \gamma$ in addition to the non-discovery of charginos and the Higgs boson at LEP which impose significant lower bounds on $m_{1 / 2}$.

In $33,34,35$, we considered the following EWPO: the $W$ boson mass, $M_{W}$, the effective weak mixing angle at the $Z$ boson resonance, $\sin ^{2} \theta_{\text {eff }}$, the width of the $Z, \Gamma_{Z}$, the Higgs mass, $m_{h}$, and $\left(g_{\mu}-2\right)$ in addtion to the BPO: $b$ decays $b \rightarrow s \gamma, B_{s} \rightarrow \mu^{+} \mu^{-}, B_{u} \rightarrow \tau \nu_{\tau}$ and the $B_{s}$ mass mixing parameter $\Delta M_{B_{s}}$. We performed the analysis of the sensitivity to $m_{1 / 2}$ moving along the WMAP strips with fixed values of $A_{0}$ and $\tan \beta$. The experimental central values, the present experimental errors and theoretical uncertainties are as described in [35. Assuming that the nine observables listed above are uncorrelated, a $\chi^{2}$ fit has been performed with

$$
\begin{aligned}
\chi^{2} \equiv & \sum_{n=1}^{7}\left[\left(\frac{R_{n}^{\exp }-R_{n}^{\text {theo }}}{\sigma_{n}}\right)^{2}+2 \log \left(\frac{\sigma_{n}}{\sigma_{n}^{\min }}\right)\right] \\
& +\chi_{m_{h}}^{2}+\chi_{B_{s}}^{2} .
\end{aligned}
$$

Here $R_{n}^{\exp }$ denotes the experimental central value of the $n$th observable $\left(M_{W}, \sin ^{2} \theta_{\text {eff }}, \Gamma_{Z},(g-2)_{\mu}\right.$ and $\left.\operatorname{BR}(b \rightarrow s \gamma), \operatorname{BR}\left(B_{u} \rightarrow \tau \nu_{\tau}\right), \Delta M_{B_{s}}\right), R_{n}^{\text {theo }}$ is the corresponding MSSM prediction and $\sigma_{n}$ denotes the combined error. Additionally, $\sigma_{n}^{\mathrm{min}}$ is the minimum combined error over the parameter space of each data set, and $\chi_{m_{h}}^{2}$ and $\chi_{B_{s}}^{2}$ denote the $\chi^{2}$ contribution coming from the experimental limits on the lightest MSSM Higgs boson mass and on $\operatorname{BR}\left(B_{s} \rightarrow \mu^{+} \mu^{-}\right)$, respectively 35 .

Details of the analysis [35] were discussed in 36. 37. and here I show the net result of the $\chi^{2}$ analysis in the CMSSM in Fig. 4 using the combined $\chi^{2}$ values for the EWPO and BPO, computed from Eq. 9, We see that the global minimum of $\chi^{2} \sim 4.5$ for both values of $\tan \beta$. This is quite a good fit for the number of experimental observables being fitted. For both values of $\tan \beta$, the focus-point region is disfavoured by comparison with the coannihilation region, though this effect is less important for $\tan \beta=50$. For $\tan \beta=10$, $m_{1 / 2} \sim 300 \mathrm{GeV}$ and $A_{0}>0$ are preferred, whereas, for $\tan \beta=50, m_{1 / 2} \sim 600 \mathrm{GeV}$ and $A_{0}<0$ are preferred. This change-over is largely due to the impact of the LEP $m_{h}$ constraint for $\tan \beta=10$ and the $b \rightarrow s \gamma$ constraint for $\tan \beta=50$.
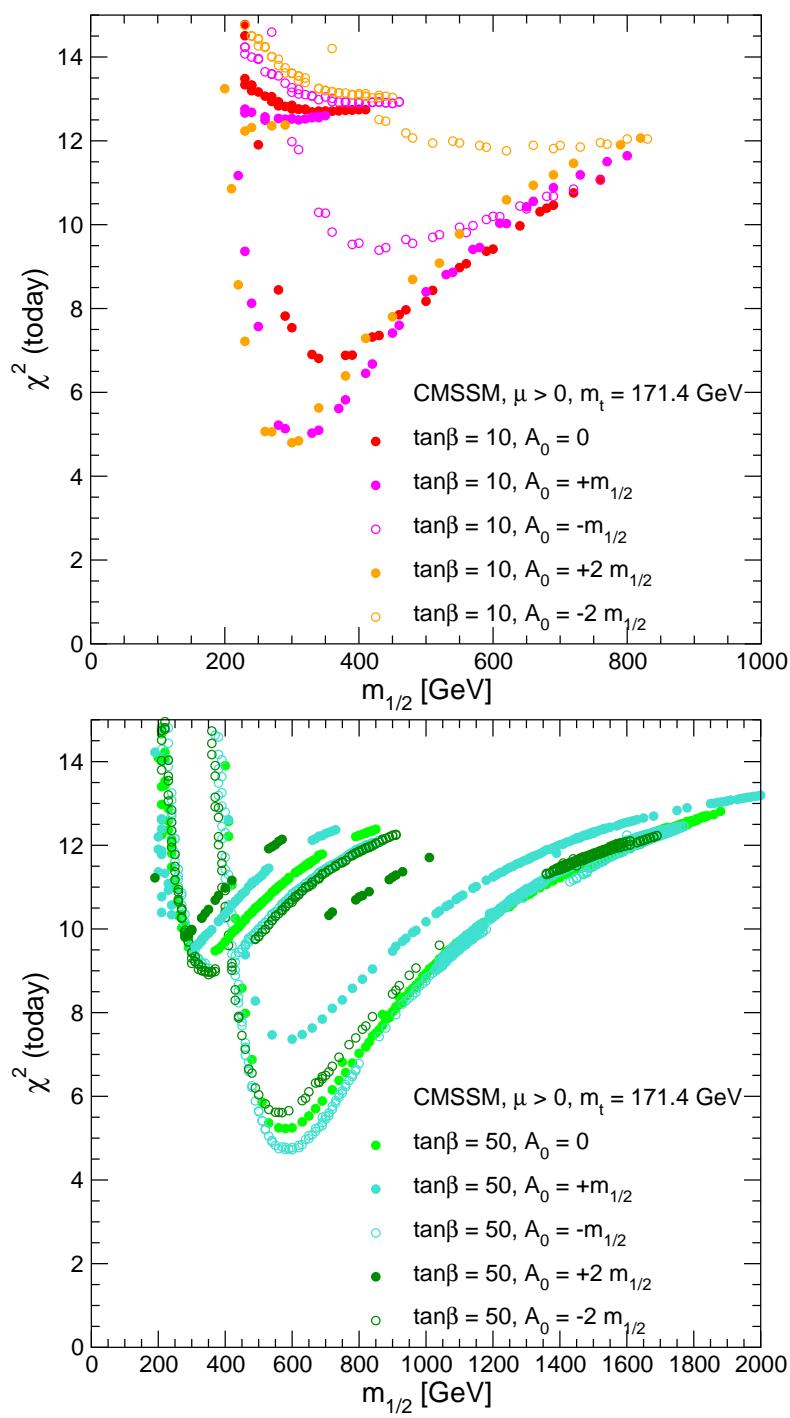

Fig. 4. The combined $\chi^{2}$ function for the electroweak observables $M_{W}, \sin ^{2} \theta_{\text {eff }}, \Gamma_{Z},(g-2)_{\mu}, m_{h}$, and the $b$ physics observables $\mathrm{BR}(b \rightarrow s \gamma), \mathrm{BR}\left(B_{s} \rightarrow \mu^{+} \mu^{-}\right), \operatorname{BR}\left(B_{u} \rightarrow\right.$ $\left.\tau \nu_{\tau}\right)$ and $\Delta M_{B_{s}}$, evaluated in the CMSSM for $\tan \beta=10$ (a) and $\tan \beta=50$ (b) for various discrete values of $A_{0}$. We use $m_{t}=171.4 \pm 2.1 \mathrm{GeV}$ and $m_{b}\left(m_{b}\right)=4.25 \pm 0.11$ $\mathrm{GeV}$, and $m_{0}$ is chosen to yield the central value of the cold dark matter density indicated by WMAP and other observations for the central values of $m_{t}$ and $m_{b}\left(m_{b}\right)$.

\section{Direct Detection in the CMSSM}

In Fig. [5 we display the expected ranges of the spinindependent cross sections in the CMSSM when we sample randomly $\tan \beta$ as well as the other CMSSM parameters 38. All points shown satisfy all phenomenological constraints and have $\Omega_{\chi} h^{2}$ less than the WMAP upper limit. Since models with low $\Omega_{\chi} h^{2}$ can not be excluded if another form of dark matter is assumed to 
exist, these models have been included, but their cross sections have been scaled by a factor $\Omega_{\chi} / \Omega_{C D M}$. In Fig. $5 \mathrm{~b}, \Sigma_{\pi N}=45 \mathrm{MeV}$ has been chosen, and in $5 \mathrm{~b}$ results for $\Sigma_{\pi N}=64 \mathrm{MeV}$ are shown for comparison. As one can see, the cross sections shift higher by a factor of a few for the larger value of $\Sigma_{\pi N}$.

Also shown on the plot are the current CDMS 39. and XENON10 [40] exclusion curves which place an upper limit on the scattering cross section. As one can see, the current limits have only just now begun to probe CMSSM models.
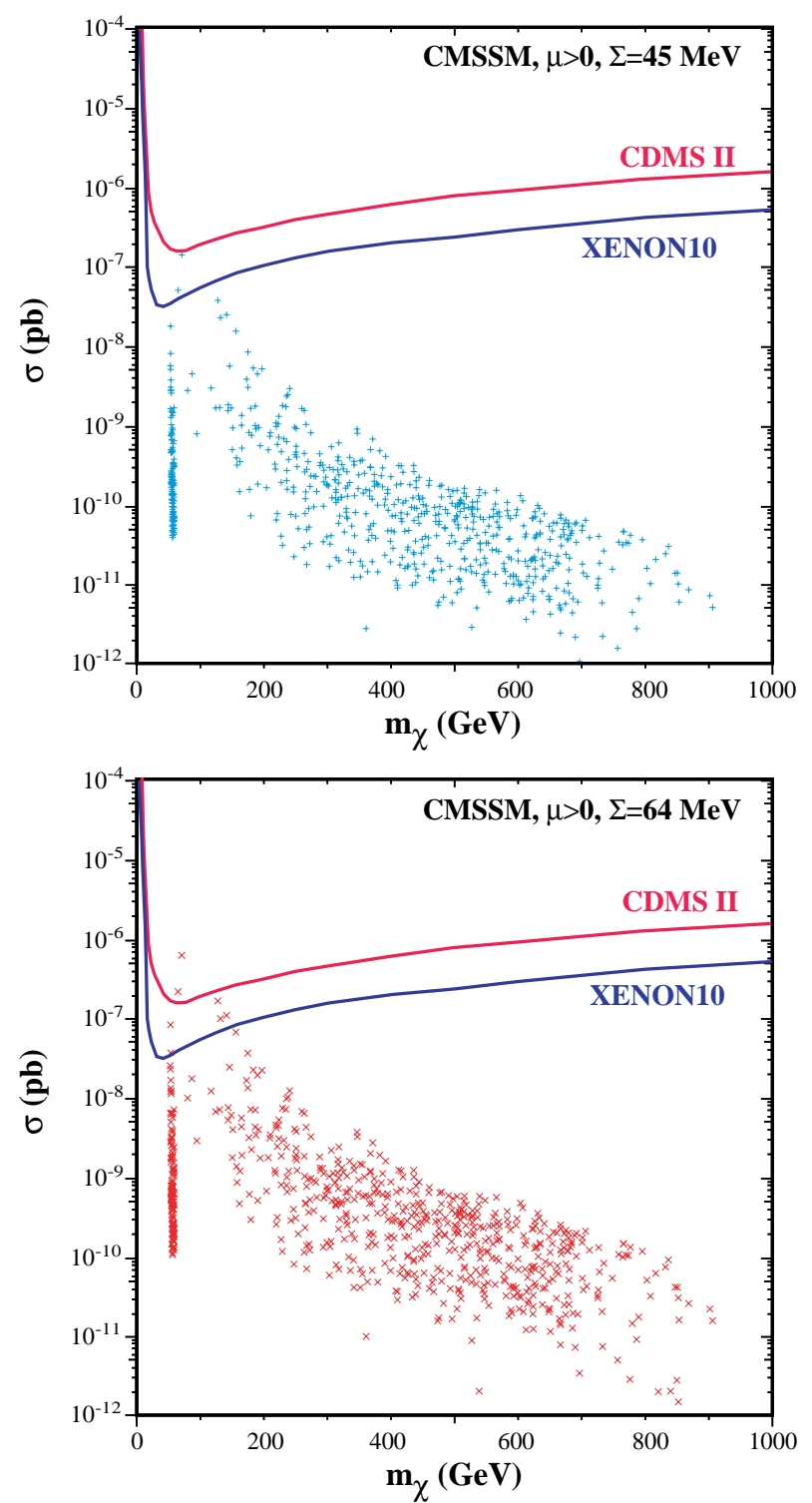

Fig. 5. Scatter plots of the spin-independent elasticscattering cross section predicted in the CMSSM for (a) $\Sigma_{\pi N}=45 \mathrm{MeV}$ and (b) $64 \mathrm{MeV}$.

\section{GUT-less models}

The input scale at which universality is assumed in CMSSM models is usually taken to be the SUSY GUT scale, $M_{G U T} \sim 2 \times 10^{16} \mathrm{GeV}$. However, it may be more appropriate in some models to assume the soft SUSYbreaking parameters to be universal at some different input scale, $M_{i n}$. Specific scenarios in which the soft SUSY-breaking parameters may be universal at a scale below $M_{G U T}$ occur in models with mixed modulusanomaly mediated SUSY breaking, also called miragemediation [41, and models with warped extra dimensions [42. In the case of mirage-mediation, the universality scale is the mirage messenger scale, which is predicted to be $M_{i n} \sim 10^{10}-10^{12} \mathrm{GeV}$ in the case of KKLT-style moduli stabilization [43]. In other models, the universality scale may lie anywhere between $1 \mathrm{TeV}$ and $M_{P l}$.

In the CMSSM with universality imposed at the GUT scale, the one-loop renormalizations of the gaugino masses $M_{a}$, where $a=1,2,3$, are the same as those for the corresponding gauge couplings, $\alpha_{a}$. Thus, at the one-loop level the gaugino masses at any scale $Q \leq M_{G U T}$ can be expressed as

$$
M_{a}(Q)=\frac{\alpha_{a}(Q)}{\alpha_{a}\left(M_{G U T}\right)} M_{a}\left(M_{G U T}\right),
$$

where $M_{a}\left(M_{G U T}\right)=m_{1 / 2}$. On the other hand, in a GUT-less CMSSM, where the gauge-coupling strengths run at all scales below the GUT scale but the soft SUSY-breaking parameters run only below the lower universality scale, $M_{i n}$, at which all the gaugino masses are assumed to be equal to $m_{1 / 2}=M_{a}\left(M_{i n}\right)$, we have

$$
M_{a}(Q)=\frac{\alpha_{a}(Q)}{\alpha_{a}\left(M_{i n}\right)} m_{1 / 2}
$$

at the one-loop level. Since the runnings of the coupling strengths in GUT and GUT-less CMSSM scenarios are identical, the low-energy effective soft gaugino masses, $M_{a}(Q)$, in GUT-less cases are less separated and closer to $m_{1 / 2}$ than in the usual GUT CMSSM. Similarly, the sfermion masses also run less and have masses closer to $m_{0}$ at the weak scale than their corresponding masses in the CMSSM.

One of the most dramatic changes when $M_{i n}$ is lowered from the GUT scale is its effect on the footprint in the $\left(m_{1 / 2}, m_{0}\right)$ plane of the constraint on the relic abundance of neutralinos inferred from WMAP. In the GUT-less CMSSM scenario [4, as the universality scale is lowered to $M_{i n} \sim 10^{12} \mathrm{GeV}$, the coannihilation strip, the funnel and the focus point regions of the CMSSM approach each other and merge, forming a small WMAP-preferred island in a sea of parameter space where the neutralino relic density is too small to provide all the cold dark matter wanted by WMAP.

As found in 44, and discussed in [45], there are already changes as the universality scale is lowered to $M_{i n}=10^{14} \mathrm{GeV}$. The allowed focus-point region starts to separate from the LEP chargino bound, moving to larger $m_{1 / 2}$. For $M_{i n}=10^{13} \mathrm{GeV}$, we found that the allowed focus-point region also dips further down, away from the electroweak vacuum condition boundary, while the coannihilation strip moves up and farther away from the region where the stau is the LSP. 

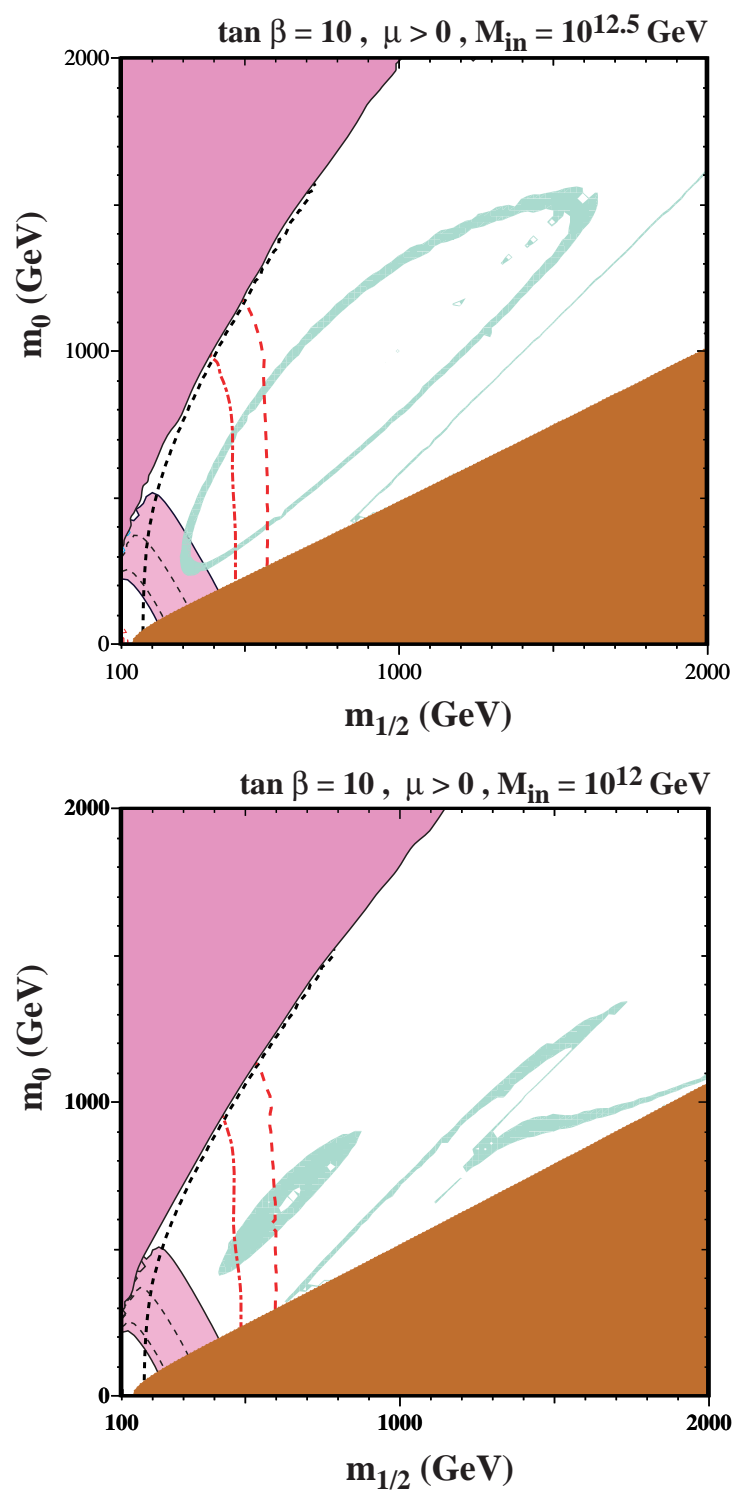

Fig. 6. Examples of $\left(m_{1 / 2}, m_{0}\right)$ planes with $\tan \beta=10$ and $A_{0}=0$ but with different values of $M_{i n}$. (a) $M_{i n}=$ $10^{12.5} \mathrm{GeV}$, (b) $M_{i n}=10^{12} \mathrm{GeV}$. In each panel, we show contours representing the LEP lower limits on the chargino mass (black dashed line), a Higgs mass of $114 \mathrm{GeV}$ (red dashed), and the more exact (and relaxed) Higgs bound (red dot-dashed). We also show the region ruled out because the $L S P$ would be charged (dark red shading), and that excluded by the electroweak vacuum condition (dark pink shading). The region favoured by WMAP has light turquoise shading, and the region suggested by $g_{\mu}-2$ at 2- $\sigma$ has medium (pink) shading, with the 1- $\sigma$ contours shown as black dashed lines.

Another remarkable feature at this value of $M_{i n}$ is the appearance of the rapid-annihilation funnel, familiar in the GUT-scale CMSSM at large $\tan \beta$, but an unfamiliar feature for $\tan \beta=10$.

As the universality scale is further decreased to $M_{\text {in }}=10^{12.5} \mathrm{GeV}$, as shown in panel (a) of Fig. 6, the atoll formed by the conjunction of what had been the focus-point and coannihilation strips has shrunk, so that it lies entirely within the range of $\left(m_{1 / 2}, m_{0}\right)$ shown in panel (a). We now see clearly two distinct regions of the plane excluded due to an excess relic density of neutralinos; the area enclosed by the atoll and the slice between the lower funnel wall and the boundary of the already-excluded $\widetilde{\tau}$-LSP region.

In panel (b) of of Fig. 6 for $M_{i n}=10^{12} \mathrm{GeV}$, the focus-point and coannihilation regions are fully combined and the atoll has mostly filled in to become a small island of acceptable relic density. To the right of this island is a strip that is provided by the lower funnel wall. The strip curves slightly as $m_{1 / 2}$ increases then takes a sharp plunge back down towards the boundary of the region where the stau is the LSP, a feature associated with the $\chi \chi \rightarrow h+A$ threshold. Reduction in the universality scale from this point results in the lower funnel wall being pushed down into the excluded $\widetilde{\tau}$ LSP region and total evaporation of the island as seen in Fig. 7 and only a small residual turquoise region at large $m_{1 / 2}$ where the relic density is within the WMAP limits. At all other points in the visible part of the $\left(m_{1 / 2}, m_{0}\right)$ plane the relic density of neutralinos is too low to provide fully the cold dark matter density preferred by WMAP et al. Of course, these SUSY models would not be excluded if there is another source of cold dark matter in the universe.

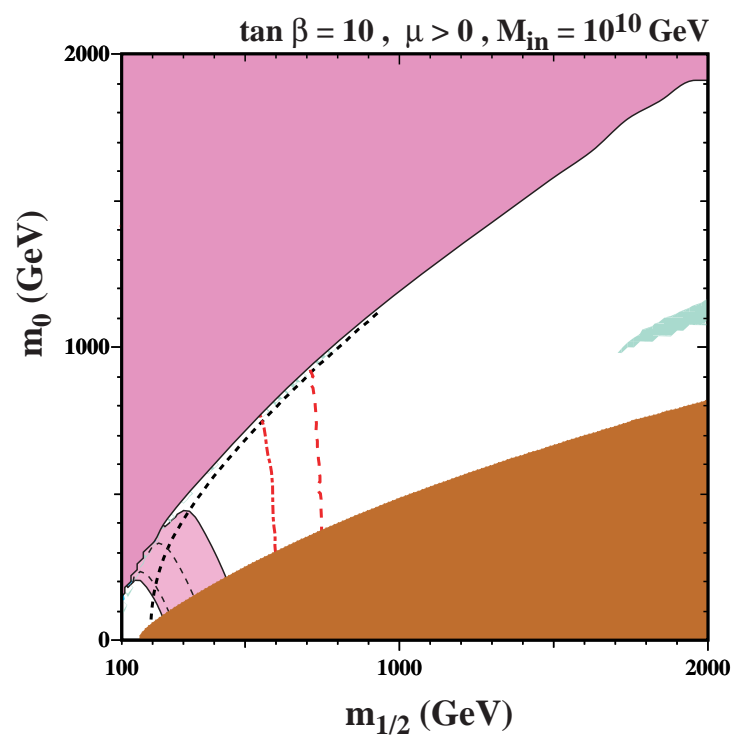

Fig. 7. As in Fig. [6 for $M_{i n}=10^{10} \mathrm{GeV}$.

\section{8 mSUGRA models}

As discussed earlier, mSUGRA models, in contrast to the CMSSM, have an additional boundary condition, namely the value of the bilinear $B$-term is fixed at the GUT scale to $B_{0}=A_{0}-m_{0}$. As a consequence, one is no longer free to choose a fixed value of $\tan \beta$ across the $m_{1 / 2}-m_{0}$ plane. Instead, $\tan \beta$ is derived for each value of $m_{1 / 2}, m_{0}$ and $A_{0}$ [16. Phenomenologically distinct planes are rather determined by a 
choice for $A_{0} / m_{0}$. In Fig. 8 , two such planes are shown with (a) $A_{0} / m_{0}=3-\sqrt{3}$ as predicted in the simplest model of supersymmetry breaking 46 and with (b) $A_{0} / m_{0}=2.0$. Shown are the contours of $\tan \beta$ (solid blue lines $)$ in the $\left(m_{1 / 2}, m_{0}\right)$ planes. Also shown are the contours where $m_{\chi^{ \pm}}>104 \mathrm{GeV}$ (near-vertical black dashed lines) and $m_{h}>114 \mathrm{GeV}$ (diagonal red dashdotted lines). The regions excluded by $b \rightarrow s \gamma$ have medium (green) shading, and those where the relic density of neutralinos lies within the WMAP range have light (turquoise) shading. The region suggested by $g_{\mu}-2$ at $2-\sigma$ has very light (pink) shading. As one can see, relatively low values of $\tan \beta$ are obtained across the planes.

Another difference between the CMSSM and models based on mSUGRA concerns the mass of the gravitino. In the CMSSM, it is not specified and and can be taken suitably large so that the neutralino is the LSP (outside the stau LSP region). In mSUGRA, the scalar masses, $m_{0}$ are determined by (and equal to) the gravitino mass. In Fig. 8, the gravitino LSP and the neutralino LSP regions are separated by dark (chocolate) solid lines. Above this line, the neutralino (or stau) is the LSP, whilst below it, the gravitino is the LSP [47. As one can see by comparing the two panels, the potential for neutralino dark matter in mSUGRA models is dependent on $A_{0} / m_{0}$. In panel (a), the only areas where the neutralino density are not too large occur where the Higgs mass is far too small or, at higher $m_{0}$ the chargino mass is too small. At larger $A_{0} / m_{0}$, the co-annihilation strip rises above the neutralinogravitino delineation. In panel (b), we see the familar co-annihilation strip. It should be noted that the focus point region is not realized in mSUGRA models as the value of $\mu$ does not decrease with increasing $m_{0}$ when $A_{0} / m_{0}$ is fixed and $B_{0}=A_{0}-m_{0}$. There are also no funnel regions, as $\tan \beta$ is never sufficiently high.

In the gravitino LSP regions, the next-to-lightest supersymmetric particle (NSP) may be either the neutralino or stau which is now unstable. The relic density of gravitinos is acceptably low only below the dashed (pink) line. This excludes a supplementary domain of the $\left(m_{1 / 2}, m_{0}\right)$ plane in panel (a). However, the strongest constraint is provided by the effect of neutralino or stau decays on big bang neucleosynthesis (BBN) 48. Outside the light (yellow) shaded region, the decays spoil the success of BBN. Note that in panel (b), there remains a region which is excluded because the stau is the LSP.

Recently, new attention has been focussed on the regions with a stau NSP due to its ability to form bound states (primarily with ${ }^{4} \mathrm{He}$ ). When such bound states occur, they catalyze certain nuclear reactions such as ${ }^{4} \mathrm{He}(\mathrm{D}, \gamma){ }^{6} \mathrm{Li}$ which is normally highly suppressed due to the production of a low energy $\gamma$ whereas the bound state reaction is not [49]. In Fig. 9a, the $\left(m_{1 / 2}, m_{0}\right)$ plane is displayed showing explicit element abundance contours [50] when the gravitino mass is $m_{3 / 2}=0.2 m_{0}$ in the absence of stau bound state effects. To the left of the solid black line the gravitino is the not the LSP. The diagonal red dotted line cor-
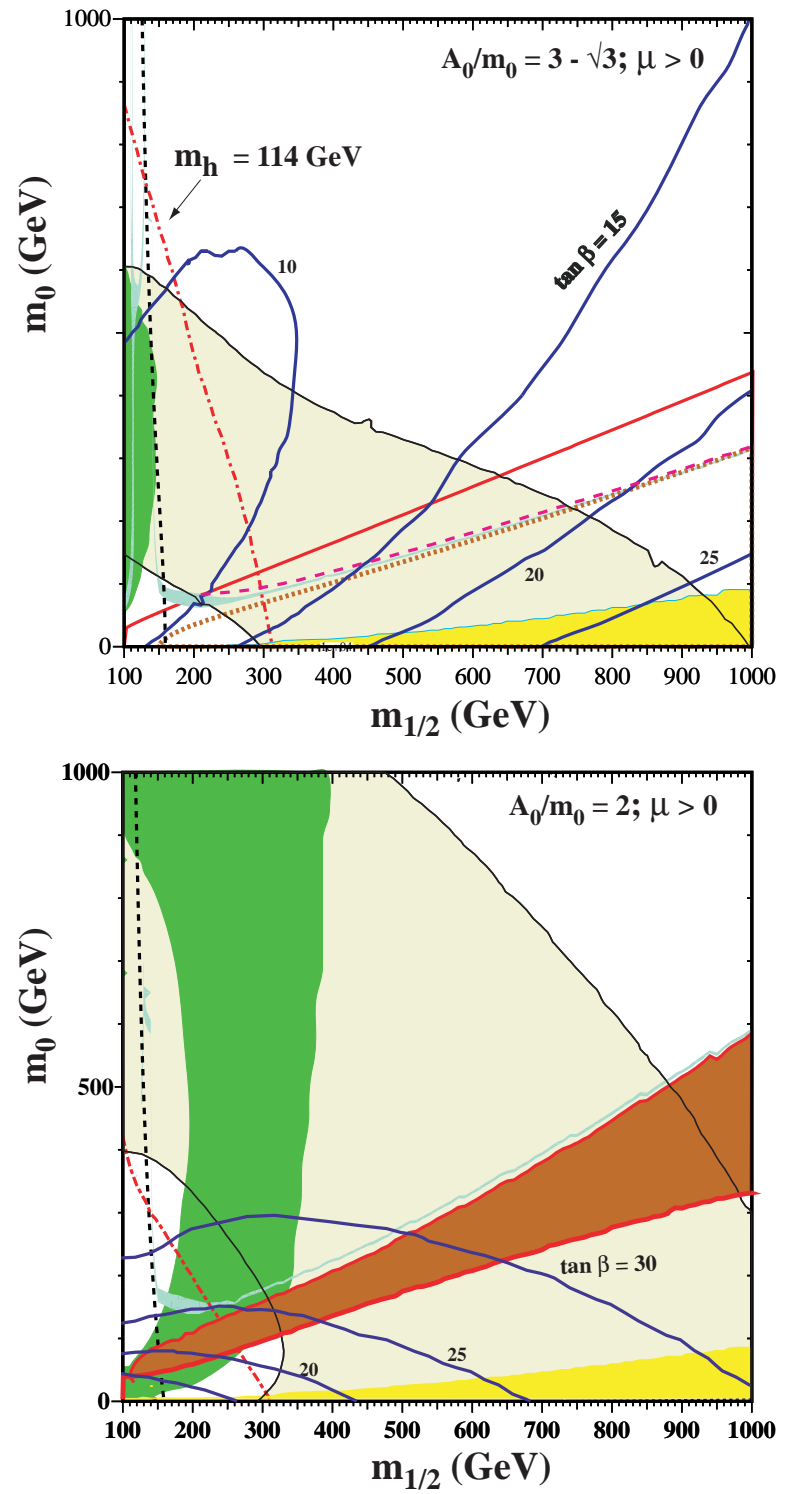

Fig. 8. Examples of $m S U G R A\left(m_{1 / 2}, m_{0}\right)$ planes with contours of $\tan \beta$ superposed, for $\mu \geq 0$ and (a) the simplest Polonyi model with $A_{0} / m_{0}=3-\sqrt{3}$, and (b) $A_{0} / m_{0}=2.0$, all with $B_{0}=A_{0}-m_{0}$. In each panel, we show the regions excluded by the LEP lower limits on MSSM particles and those ruled out by $b \rightarrow s \gamma$ decay (medium green shading): the regions favoured by $g_{\mu}-2$ are very light (yellow) shaded, bordered by a thin (black) line. The dark (chocolate) solid lines separate the gravitino LSP regions. The regions favoured by WMAP in the neutralino LSP case have light (turquoise) shading. The dashed (pink) line corresponds to the maximum relic density for the gravitino $L S P$, and regions allowed by BBN constraint neglecting the effects of bound states on NSP decay are light (yellow) shaded.

responds to the boundary between a neutralino and stau NSP. Above the line, the neutralino is the NSP, and below it, the NSP is the stau. Very close to this boundary, there is a diagonal brown solid line. Above this line, the relic density of gravitinos from NSP decay 
is too high, i.e.,

$$
\frac{m_{3 / 2}}{m_{N S P}} \Omega_{N S P} h^{2}>0.12 .
$$

Thus we should restrict our attention to the area below this line.

The very thick green line labelled ${ }^{7} \mathrm{Li}=4.3$ corresponds to the contour where ${ }^{7} \mathrm{Li} / \mathrm{H}=4.3 \times 10^{-10}$, a value very close to the standard $B B N$ result for ${ }^{7} \mathrm{Li} / \mathrm{H}$. It forms a ' $\mathrm{V}$ ' shape, whose right edge runs along the neutralino-stau NSP border. Below the V, the abundance of ${ }^{7} \mathrm{Li}$ is smaller than the standard $\mathrm{BBN}$ result. However, for relatively small values of $m_{1 / 2}$, the ${ }^{7} \mathrm{Li}$ abundance does not differ very much from this standard BBN result: it is only when $m_{1 / 2} \gtrsim$ $3000 \mathrm{GeV}$ that ${ }^{7} \mathrm{Li}$ begins to drop significantly. The stau lifetime drops with increasing $m_{1 / 2}$, and when $\tau \sim 1000 \mathrm{~s}$, at $m_{1 / 2} \sim 4000 \mathrm{GeV}$, the ${ }^{7} \mathrm{Li}$ abundance has been reduced to an observation-friendly value close to $2 \times 10^{-10}$ as claimed in [51] and shown by the (unlabeled) thin dashed (green) contours.

The region where the ${ }^{6} \mathrm{Li} /{ }^{7} \mathrm{Li}$ ratio lies between 0.01 and 0.15 forms a band which moves from lower left to upper right. As one can see in the orange shading, there is a large region where the lithium isotopic ratio can be made acceptable. However, if we restrict to $\mathrm{D} / \mathrm{H}$ $<4.0 \times 10^{-5}$, we see that this ratio is interesting only when ${ }^{7} \mathrm{Li}$ is at or slightly below the standard BBN result.

Turning now to Fig. 9b, we show the analogous results when the bound-state effects are included in the calculation. The abundance contours are identical to those in panel (a) above the diagonal dotted line, where the NSP is a neutralino and bound states do not form. We also note that the bound state effects on $\mathrm{D}$ and ${ }^{3} \mathrm{He}$ are quite minimal, so that these element abundances are very similar to those in Fig. 9a. However, comparing panels (a) and (b), one sees dramatic bound-state effects on the lithium abundances. Everywhere to the left of the solid blue line labeled 0.15 is excluded. In the stau NSP region, this means that $m_{1 / 2} \gtrsim 1500 \mathrm{GeV}$. Moreover, in the stau region to the right of the ${ }^{6} \mathrm{Li} /{ }^{7} \mathrm{Li}=0.15$ contour, the ${ }^{7} \mathrm{Li}$ abundance drops below $9 \times 10^{-11}$ (as shown by the thin green dotted curve). In this case, not only do the bound-state effects increase the ${ }^{6} \mathrm{Li}$ abundance when $m_{1 / 2}$ is small (i.e., at relatively long stau lifetimes), but they also decrease the ${ }^{7} \mathrm{Li}$ abundance when the lifetime of the stau is about $1500 \mathrm{~s}$. Thus, at $\left(m_{1 / 2}, m_{0}\right) \simeq(3200,400)$, we find that ${ }^{6} \mathrm{Li} /{ }^{7} \mathrm{Li} \simeq 0.04,{ }^{7} \mathrm{Li} / \mathrm{H} \simeq 1.2 \times 10^{-10}$, and $\mathrm{D} / \mathrm{H} \simeq 3.8 \times 10^{5}$. Indeed, when $m_{1 / 2}$ is between $3000-4000 \mathrm{GeV}$, the bound state effects cut the ${ }^{7} \mathrm{Li}$ abundance roughly in half. In the darker (pink) region, the lithium abundances match the observational plateau values, with the properties ${ }^{6} \mathrm{Li} /{ }^{7} \mathrm{Li}>0.01$ and $0.9 \times 10^{-10}<{ }^{7} \mathrm{Li} / \mathrm{H}<2.0 \times 10^{-10}$.

Next we return to an example of an mSUGRA model based on the Polonyi model for which $A_{0} / m_{0}=$ $3-\sqrt{3}$ with the condition that $m_{3 / 2}=m_{0}$. In Fig. 10a, we show the mSUGRA model without the bound states. In the upper part of the plane, we do not have gravitino
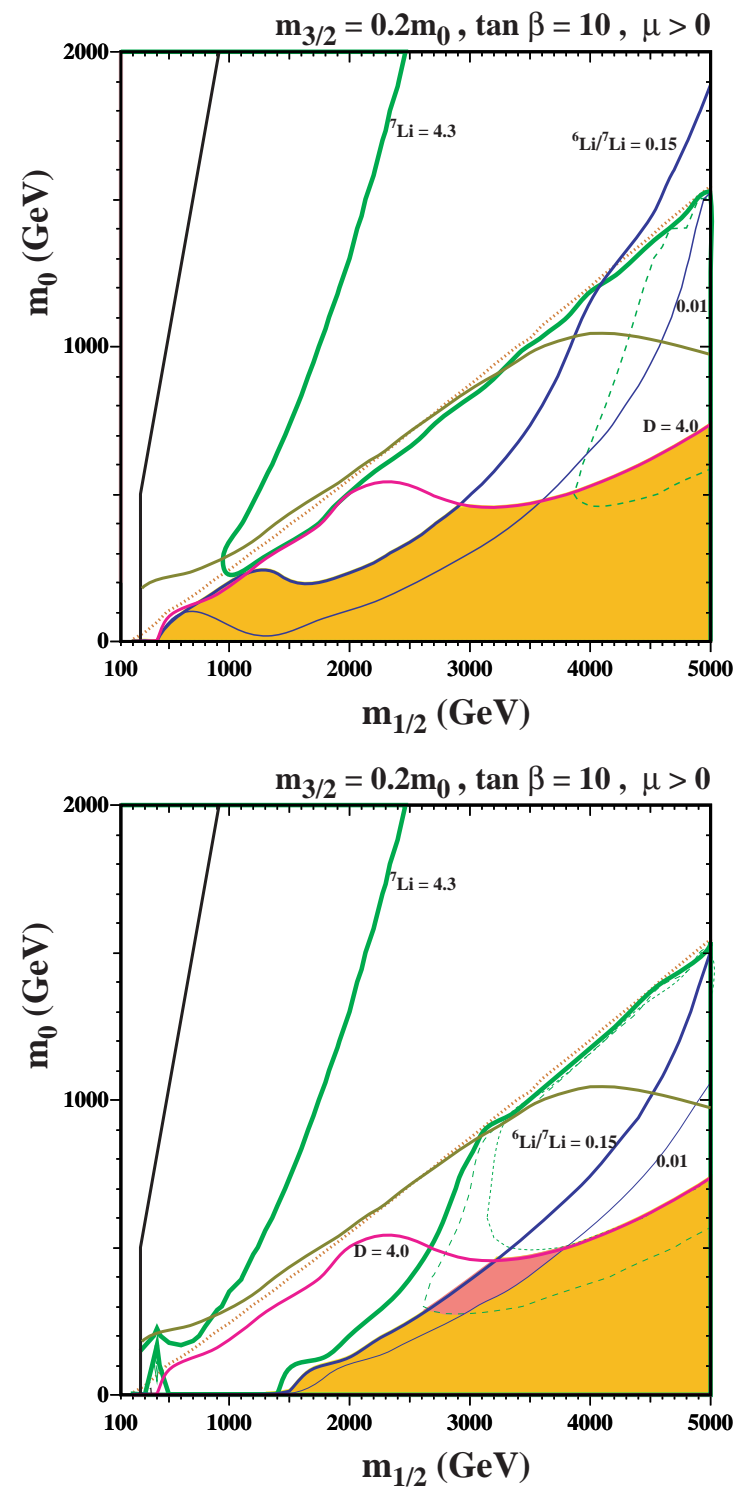

Fig. 9. $A n\left(m_{1 / 2}, m_{0}\right)$ planes for $A_{0}=0, \mu>0$ and $\tan \beta=10$ with $m_{3 / 2}=0.2 m_{0}$ (a) without and (b) with the effects of boundstates included. The regions to the left of the solid black lines are not considered, since there the gravitino is not the LSP. In the orange (light) shaded regions, the differences between the calculated and observed light-element abundances are no greater than in standard BBN without late particle decays. In the pink (dark) shaded region in panel $b$, the abundances lie within the ranges favoured by observation. The significances of the other lines and contours are explained in the text.

dark matter. We see that ${ }^{3} \mathrm{He} / \mathrm{D}[52$ eliminates all but a triangular area which extends up to $m_{0}=1000 \mathrm{GeV}$, when $m_{1 / 2}=5000 \mathrm{GeV}$. Below the ${ }^{3} \mathrm{He} / \mathrm{D}=1$ contour, $\mathrm{D}$ and ${ }^{7} \mathrm{Li}$ are close to their standard $\mathrm{BBN}$ values, and there is a substantial orange shaded region. We note that ${ }^{6} \mathrm{Li}$ is interestingly high, between 0.01 and 0.15 in much of this region.

As seen in Fig. 10p, when bound-state effects are included in this mSUGRA model, both lithium isotope abundances are too large except in the extreme lower 
right corner, where there is a small region shaded orange. However, there is no region where the lithium abundances fall within the favoured plateau ranges.
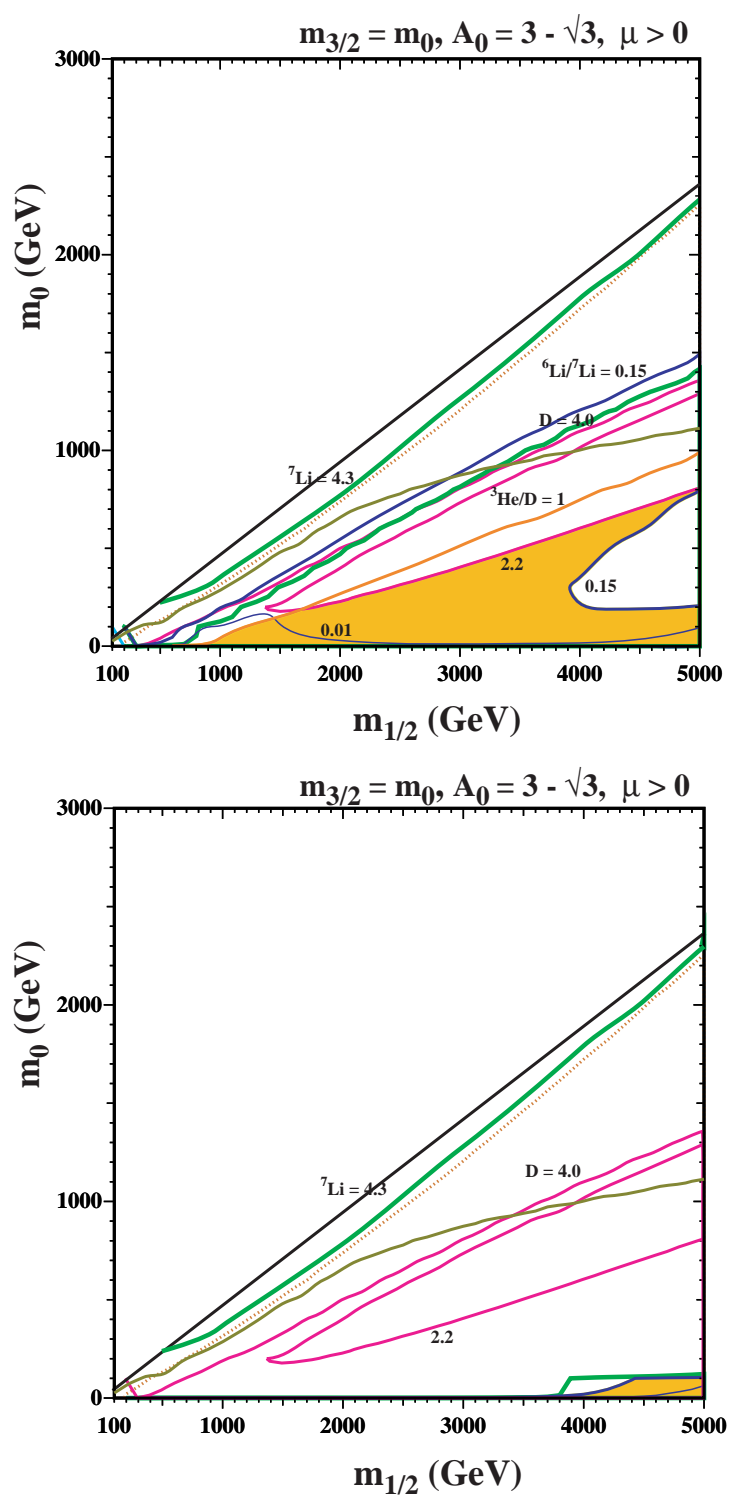

Fig. 10. As in Fig. 9, for the mSUGRA case with $A_{0} / m_{0}=3-\sqrt{3}$.

The prospects for direct detection in the mSUGRA models (when the neutralino is the LSP) is very similar to that of the CMSSM but slightly more difficult. In a plot such as that shown in Fig. 5, the mSUGRA predictions are down by a factor of about 2 or less.

\section{The NUHM}

In the NUHM, the Higgs soft masses are treated independently from $m_{0}$. In effect, this allows one to choose $\mu$ and the Higgs pseudoscalar mass, $m_{A}$ freely (up to phenomenological constraints). Two examples of planes in the NUHM are shown in Fig. 11, In panel (a), an $m_{1 / 2}-m_{0}$ plane is shown with $\mu=700 \mathrm{GeV}$ and $m_{A}=$ $400 \mathrm{GeV}$ fixed across the plane [18]. As usual, the light (turquoise) shaded area is the cosmologically preferred region. There is a bulk region satisfying this preference at $m_{1 / 2} \sim 150 \mathrm{GeV}$ to $350 \mathrm{GeV}$ and $m_{0} \sim 100 \mathrm{GeV}$. The dark (red) shaded regions are excluded because a charged sparticle is lighter than the neutralino. As in the CMSSM, there are light (turquoise) shaded strips close to these forbidden regions where coannihilation suppresses the relic density sufficiently to be cosmologically acceptable. Further away from these regions, the relic density is generally too high. At small $m_{1 / 2}$ and $m_{0}$ the left handed sleptons, and also the sneutrinos, become lighter than the neutralino. The darker (dark blue) shaded area is where a sneutrino is the LSP.

The near-vertical dark (black) dashed and light (red) dot-dashed lines in Fig. 11 are the LEP exclusion contours $m_{\chi^{ \pm}}>104 \mathrm{GeV}$ and $m_{h}>114 \mathrm{GeV}$ respectively. As in the CMSSM case, they exclude low values of $m_{1 / 2}$, and hence rule out rapid relic annihilation via direct-channel $h$ and $Z^{0}$ poles. The solid lines curved around small values of $m_{1 / 2}$ and $m_{0}$ bound the light (pink) shaded region favoured by $a_{\mu}$ and recent analyses of the $e^{+} e^{-}$data.

A striking feature in Fig. 11(a) when $m_{1 / 2} \sim 450$ $\mathrm{GeV}$ is a strip with low $\Omega_{\chi} h^{2}$, which has bands with acceptable relic density on either side. The low- $\Omega_{\chi} h^{2}$ strip is due to rapid annihilation via the direct-channel $A, H$ poles which occur when $m_{\chi}=m_{A} / 2=200 \mathrm{GeV}$, indicated by the near-vertical solid (blue) line. These were found in the CMSSM 10,12, but at larger $\tan \beta$. At higher $m_{1 / 2} \sim 1300-1400 \mathrm{GeV}$, there is another region of acceptable relic density. This band, the transition band, is broadened because the neutralino acquires significant Higgsino content as $m_{1 / 2}$ becomes greater than $\mu$, and the relic density is suppressed by the increased $W^{+} W^{-}$production. To the right of this band, the relic density falls below the WMAP value.

Another example of an NUHM plane is shown in panel (b) of Fig. 11, representing an $\mu-m_{A}$ plane with a fixed choice of values of $\tan \beta=35, m_{1 / 2}=500$ $\mathrm{GeV}, m_{0}=1000 \mathrm{GeV}$ and $A_{0}=038$. Line types and shading are as in panel (a). The region with acceptable relic density now takes the form of a narrow strip of values of $\mu \sim \pm 300 \rightarrow 350 \mathrm{GeV}$ where the relic density lies within the WMAP range for almost all values of $m_{A}$. The exception is a narrow strip centred on $m_{A} \sim 430 \mathrm{GeV}$, namely the rapid-annihilation funnel where $m_{\chi} \sim m_{A} / 2$, which would be acceptable if there is some other source of cold dark matter. Note the increased importance of the $b \rightarrow s \gamma$ constraint which now excludes almost all of $\mu<0$.

By using the properties found in the $\mu-m_{A}$ plane discussed above, one can construct a $m_{A}-\tan \beta$ plane such that (nearly) the entire plane respects the cosmological relic density constraint [35,53. These plane can be used as a particularly useful benchmark for existing and future searchs for Higgs bosons [53. An example of such a plane is shown in Fig. 12 with fixed $m_{1 / 2}=500$ $\mathrm{GeV}$ and $m_{0}=1000 \mathrm{GeV}$. As one can see, the light shaded (turquoise) region now fills up the plane ex- 

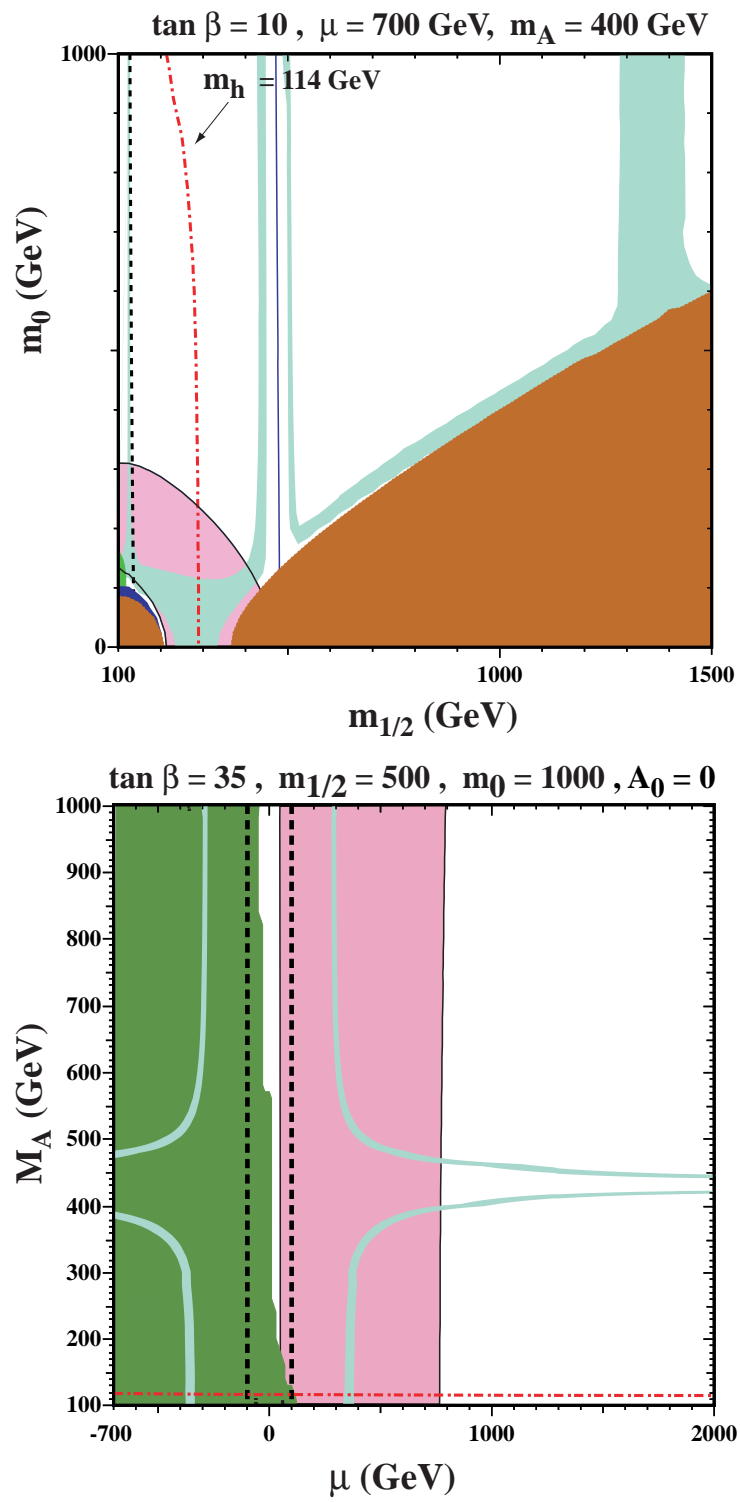

Fig. 11. Projections of the NUHM model on (a) the $\left(m_{1 / 2}, m_{0}\right)$ planes for $\tan \beta=10$ and $\mu=700 \mathrm{GeV}$ and $m_{A}=400 \mathrm{GeV}$ and $(b)$ the $\mu-m_{A}$ plane with $m_{1 / 2}=500$ GeV, $m_{0}=1000 \mathrm{GeV}, \tan \beta=35$ and $A_{0}=0$. The (red) dot-dashed lines are the contours $m_{h}=114 \mathrm{GeV}$ as calculated using FeynHiggs [24], and the near-vertical (black) dashed lines are the contours $m_{\chi^{ \pm}}=104 \mathrm{GeV}$. The light (turquoise) shaded areas are the cosmologically preferred regions. The dark (brick red) shaded regions is excluded because a charged particle is lighter than the neutralino, and the darker (dark blue) shaded regions is excluded because the LSP is a sneutrino. In panel (a) there is a very small medium (green) shaded region excluded by $b \rightarrow s \gamma$, at small $m_{1 / 2}$. In panel (b), it excluded most of $\mu<0$. The regions allowed by the E821 measurement of $a_{\mu}$ at the 2- $\sigma$ level, are shaded (pink) and bounded by solid black lines.

cept for a vertical swath where s-channel annihilation through $m_{A}$ drives the relic density to small values. A likelihood analysis of this NUHM benchmark surface, including the EWPO $M_{W}, \sin ^{2} \theta_{\text {eff }}, \Gamma_{Z},(g-2)_{\mu}$ and $m_{h}$ and the $\mathrm{BPO} \operatorname{BR}(b \rightarrow s \gamma), \operatorname{BR}\left(B_{s} \rightarrow \mu^{+} \mu^{-}\right)$,
$\operatorname{BR}\left(B_{u} \rightarrow \tau \nu_{\tau}\right)$ and $\Delta M_{B_{s}}$ was performed recently in [35. The lowest $\chi^{2}$ value in this plane is $\chi_{\min }^{2}=7.4$ and the corresponding best-fit point is shown by a (red) cross as are the $\Delta \chi^{2}=2.30$ and 4.61 contours around the best-fit point. The (black shaded) region in the lower left is excluded at the $95 \%$ C.L. by the LEP Higgs searches in the channel $e^{+} e^{-} \rightarrow Z^{*} \rightarrow$ $Z h, H[23]$.

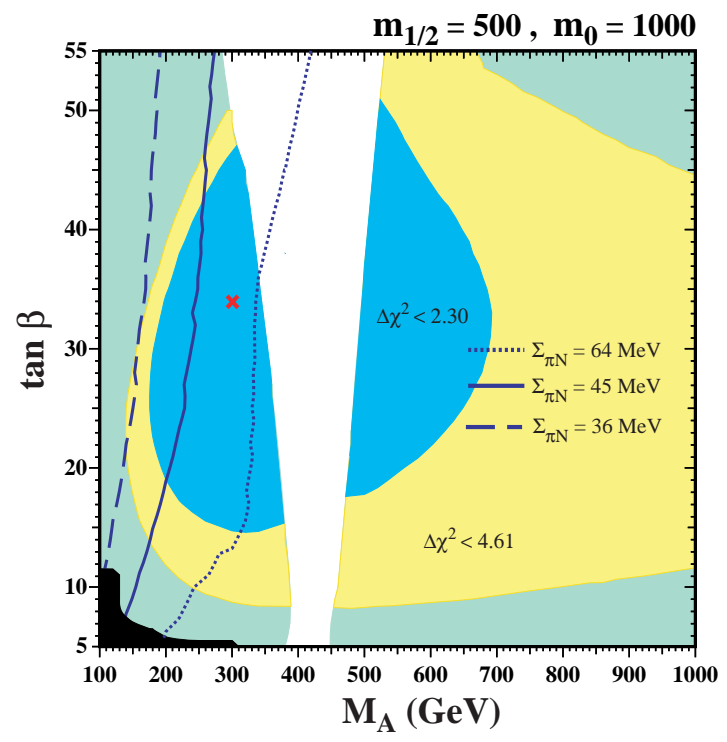

Fig. 12. The $\left(m_{A}, \tan \beta\right)$ plane for the NUHM benchmark surface displaying the contours of $\Delta \chi^{2}$ found in a recent global fit to EWPO and BPO [35]. Also displayed is the expected sensitivity of present direct searches for the scattering of dark matter particles.

Also shown in Fig. 11 is the current reach of the XENON10 experiment 40 . The solid line in the figure correspond to the XENON10 bound obtained assuming $\Sigma_{\pi N}=45 \mathrm{MeV}$. The corresponding reach obtained if $\Sigma_{\pi N}=64 \mathrm{MeV}$ is shown by the dotted curve. As one can see, in this case, the best fit point would already have been probed by the XENON10 experiment. If the strangeness content of the proton were zero, it would correspond to $\Sigma_{\pi N}=36 \mathrm{MeV}$, and the XENON10 reach in this case is displayed by the dashed curve. Finally, we note that a future sensitivity to a cross section of $10^{-8} \mathrm{pb}$ would cover the entire surface.

As can be inferred from the benchmark surface shown above, in the NUHM, current constraints already exclude many interesting models [38. These models generically have large scattering cross sections. Scatter plots of showing the reach of CDMS and XENON10 for both $\Sigma_{\pi N}=45 \mathrm{MeV}$ and $64 \mathrm{MeV}$ are shown in Fig. 13. In comparison with Fig. 5 , one sees that many more viable models are already currently being probed.

\section{A Hint of Higgs}

The CDF and D0 Collaborations have already established important limits on the (heavier) MSSM Higgs 

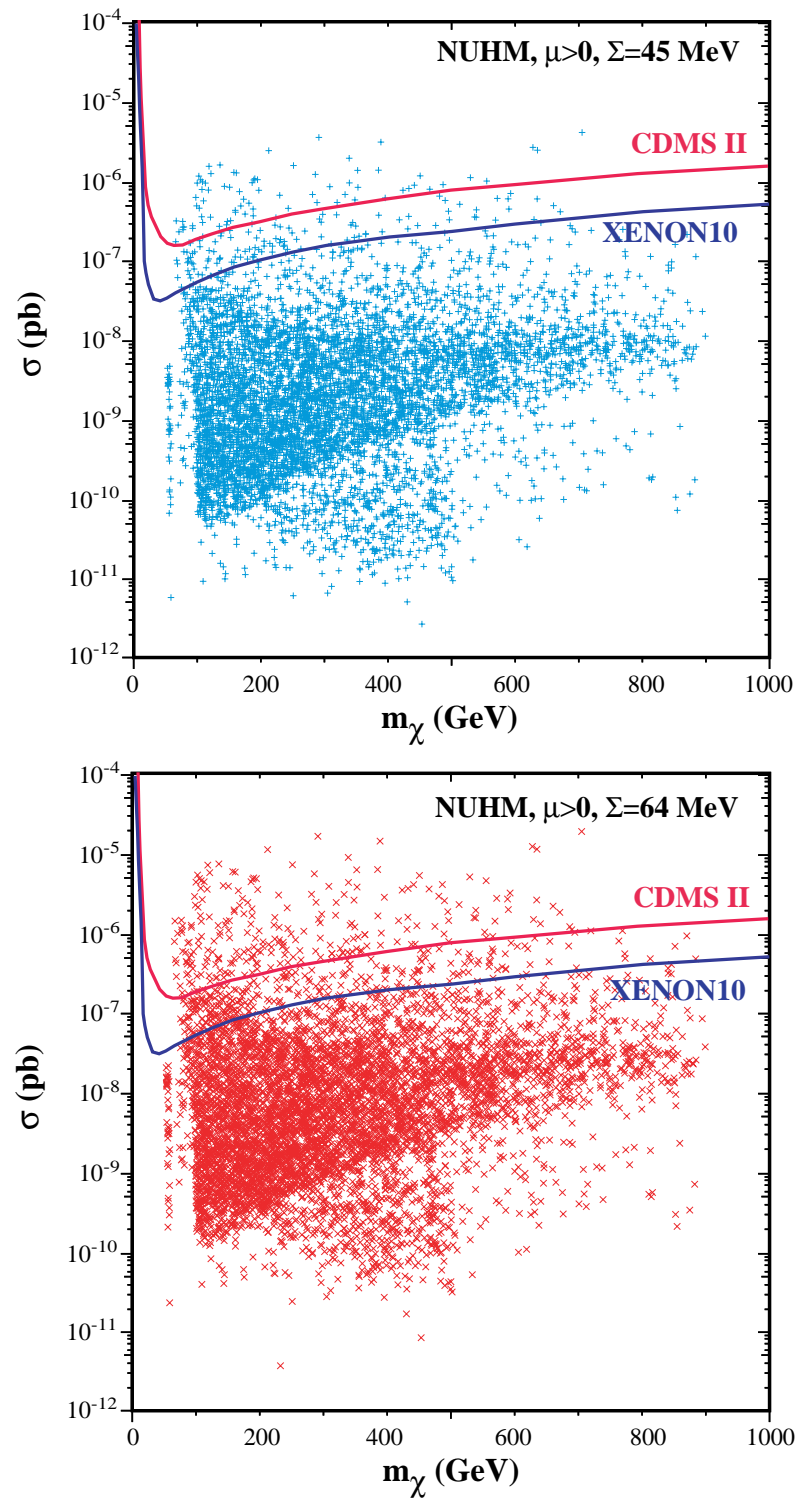

Fig. 13. Scatter plots of the spin-independent elasticscattering cross section predicted in the CMSSM (a) for $\Sigma_{\pi N}=45 \mathrm{MeV}($ b) and $64 \mathrm{MeV}$.

bosons, particularly at large $\tan \beta$ [54,55, 56, 57, 58. Recently the CDF Collaboration, investigating the channel

$$
p \bar{p} \rightarrow \phi \rightarrow \tau^{+} \tau^{-}, \quad(\phi=h, H, A),
$$

has been unable to improve these limits to the extent of the sensitivity expected with the analyzed integrated luminosity of $\sim 1 \mathrm{fb}^{-1}$ [57], whereas there is no indication of any similar effect in D0 data 58. Within the MSSM the channel (13) is enhanced as compared to the corresponding SM process by roughly a factor of $\tan ^{2} \beta /\left(\left(1+\Delta_{b}\right)^{2}+9\right)$ [59], where $\Delta_{b}$ includes loop corrections to the $\phi b \bar{b}$ vertex (see [59] for details) and is subdominant for the $\tau^{+} \tau^{-}$final state. Correspondingly, the unexpected weakness of the CDF exclusion might be explicable within the MSSM if $m_{A} \approx$ $160 \mathrm{GeV}$ and $\tan \beta \gtrsim 45$.
In the CMSSM, there are no parameter sets consistent with all the experimental and theoretical constraints. In particular, low values of $m_{1 / 2}$ are required to obtain low values of $m_{A}$. For $m_{A} \simeq 160 \mathrm{GeV}$ and the other parameters tuned to fulfill the $B$ physics constraints, there are no CMSSM solutions for which the lightest Higgs mass is not in direct contradiction with experimental limits 23]. However, we do find that all the constraints may be satisfied in the NUHM 60, 61.

We consider first the $\left(m_{1 / 2}, m_{0}\right)$ plane shown in panel (a) of Fig. 14, which has $\tan \beta=45, \mu=370 \mathrm{GeV}$ and $A_{0}=-1800 \mathrm{GeV}$, as well as $M_{A}=160 \mathrm{GeV}$. As before, the dark (brown) shaded region at low $m_{0}$ is forbidden, because there the LSP would be the lighter stau. The WMAP cold dark matter constraint is satisfied only within the lighter (turquoise) shaded region. To the left of this region, the relic density is too small, due to $s$-channel annihilation through the Higgs pseudoscalar $A$. As $m_{1 / 2}$ increases away from the pole, the relic density increases toward the WMAP range. However, as $m_{1 / 2}$ is increased, the neutralino acquires a larger Higgsino component and annihilations to pairs of $W$ and $Z$ bosons become enhanced. To the right of this transition region, the relic density again lies below the WMAP preferred value. The shaded region here is therefore an overlap of the funnel and transition regions discussed in [18. The $\operatorname{BR}\left(B_{s} \rightarrow \mu^{+} \mu^{-}\right)$constraint 62 is satisfied between the outer black dashdotted lines, labelled $10^{-7}$, representing the current limit on that branching ratio. Also shown are the contours where the branching ratio is $2 \times 10^{-8}$, close to the sensitivity likely to be attainable soon by CDF and D0. Between these two contours, there is a strong cancellation between the flavor-violating contributions arising from the Higgs and chargino couplings at the one-loop level and the Wilson coefficient counterterms contributing to $\operatorname{BR}\left(B_{s} \rightarrow \mu^{+} \mu^{-}\right)[63$.

The dash-dotted (red) line shows the contour corresponding to $m_{h}=114 \mathrm{GeV}$, and only the region to the right of this line is compatible with the constraint imposed by $m_{h}$ (it should be kept in mind that there is still a $\sim 3 \mathrm{GeV}$ uncertainty in the prediction of $m_{h}$ 24). Also shown in pink shading is the region favoured by $(g-2)_{\mu}$ at the two- $\sigma$ level. The one- and two- $\sigma$ contours for $(g-2)_{\mu}$ are shown as elliptical dashed and solid black contours, respectively. The region which is compatible with the WMAP relic density and $m_{h}$, and is also within the two- $\sigma(g-2)_{\mu}$ experimental bound, has $\operatorname{BR}\left(B_{s} \rightarrow \mu^{+} \mu^{-}\right)>2 \times 10^{-8}$. The measured value of $\operatorname{BR}(b \rightarrow s \gamma)$ is in agreement with the theory prediction only to the left of the solid (green) region.

We see that there is a narrow wedge of allowed parameter space in 14(a), which has $m_{1 / 2} \sim 600 \mathrm{GeV}$ and $m_{0} \sim 700$ to $1100 \mathrm{GeV}$. The $\mathrm{BR}(b \rightarrow s \gamma)$ constraint is satisfied easily throughout this region, and $(g-2)_{\mu}$ cuts off the top of the wedge, which would otherwise have extended to $m_{0} \gg 1500 \mathrm{GeV}$. Within the allowed wedge, $m_{h}$ is very close to the LEP lower limit, and $\operatorname{BR}\left(B_{s} \rightarrow \mu^{+} \mu^{-}\right)>2 \times 10^{-8}$. If $M_{A}$ were much smaller $(<130 \mathrm{GeV})$, there would be no wedge consis- 

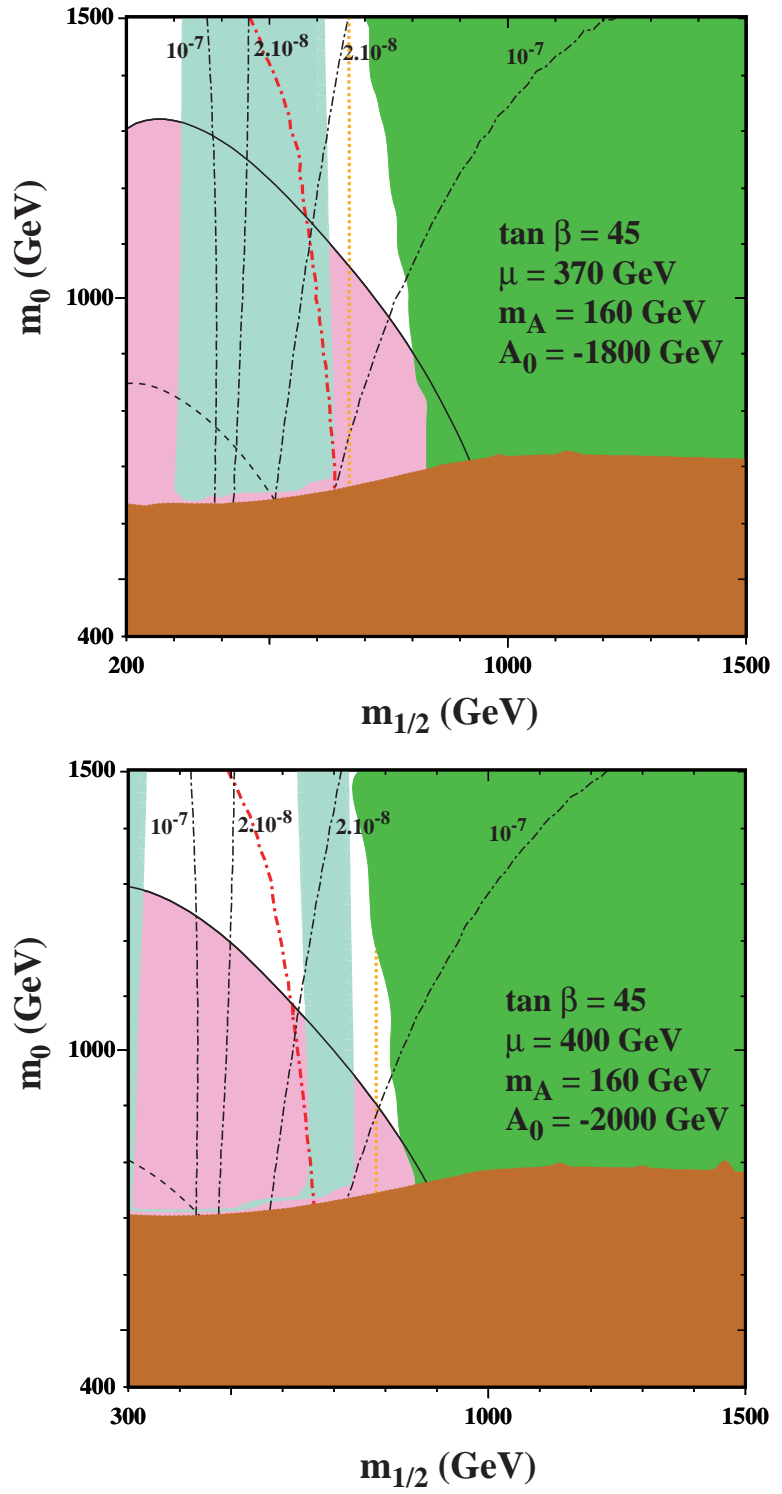

Fig. 14. The NUHM parameter space as a function of $m_{1 / 2}$ and $m_{0}$ for $\mu=370(400) \mathrm{GeV}$ and $A_{0}=$ $-1800(-2000) \mathrm{GeV}$ in panels a (b). We fix $M_{A}=160 \mathrm{GeV}$, $\tan \beta=45, m_{t}=171.4 \mathrm{GeV}$ and $m_{b}=4.25 \mathrm{GeV}$. For the description of the various lines and shaded areas, see the text.

tent simultaneously with the $\Omega_{\mathrm{CDM}}, m_{h}$ and $\mathrm{BR}\left(B_{s} \rightarrow\right.$ $\left.\mu^{+} \mu^{-}\right)$constraints.

Given the matrix element uncertainties for direct detection summarized above, we show in 14a) the 1- $\sigma$ lower limit on the calculated value of the elastic cross section as compared to the CDMS upper limit [39]. In the portion of the plane to the left of the (orange) dotted line, the lower limit on the calculated spinindependent elastic cross section is smaller than the CDMS upper bound, assuming the canonical local density. Whilst we have assumed $\Sigma_{\pi N}=45 \mathrm{MeV}$, the calculated lower limit effectively assumes zero strangeness contribution to the proton mass, i.e., $y=0$. In the region of interest, the lower limit on the calculated cross section is about $80 \%$ of the CDMS upper bound, whereas with a strangeness contribution of $y=0.2$, the cross section would exceed the CDMS bound by a factor of $\sim 3$. Thus, if Nature has picked this corner of the NUHM parameter space, we expect direct detection of dark matter to be imminent. Consistency with the XENON10 limit would further require a reduction in the local dark matter density (to its lower limit). Intriguingly, the XENON10 experiment has seen some potential signal events that are, however, interpreted as background.

Panel (a) of 15 explores the $\left(\mu, A_{0}\right)$ plane for the choice $\left(m_{1 / 2}, m_{0}\right)=(600,800) \mathrm{GeV}$, values close to the lower tip of the allowed wedge in 14(a). In this case, the region allowed by the $\operatorname{BR}\left(B_{s} \rightarrow \mu^{+} \mu^{-}\right)$constraint is below the upper dash-dotted black line, and the LEP $m_{h}$ constraint is satisfied only above the dash-dotted red line. We see that only a restricted range $360 \mathrm{GeV}<$ $\mu<390 \mathrm{GeV}$ is compatible with the dark matter constraint. This corresponds to the transition strip where the neutralino is the appropriate bino/Higgsino combination. To the left of this strip, the relic density is too small and to the right, it is too large. Only a very restricted range of $A_{0} \sim-1600 \mathrm{GeV}$ is compatible simultaneously with the $m_{h}$ and $\operatorname{BR}\left(B_{s} \rightarrow \mu^{+} \mu^{-}\right)$constraints. Very large negative values of $A_{0}$ are excluded as the LSP is the lighter stau. On the $\left(\mu, A_{0}\right)$ plane, the elastic scattering cross section is a rapidly decreasing function of $\mu$ and is almost independent of $A_{0}$. Indeed, NUHM points excluded by CDMS (or XENON10) generally have low values of $\mu$ and $M_{A}$ [13. Values of $\mu>$ $355 \mathrm{GeV}$ are compatible with CDMS if the strangeness contribution to the proton mass is negligible. For this choice of parameters, the entire displayed plane is compatible with $\operatorname{BR}(b \rightarrow s \gamma)$ and $(g-2)_{\mu}$.

Panel (b) of Fig. 15 shows what happens if $m_{1 / 2}$ is increased to $700 \mathrm{GeV}$, keeping $m_{0}$ and the other inputs the same. Compared to Fig. 15(a), we see that the WMAP strip becomes narrower and shifts to larger $\mu \sim 400 \mathrm{GeV}$, and that $\mathrm{BR}(b \rightarrow s \gamma)$ starts to exclude a region visible at smaller $\mu$. If $m_{1 / 2}$ were to be increased much further, the dark matter constraint and $\mathrm{BR}(b \rightarrow s \gtrsim)$ would no longer be compatible for this value of $m_{0}$. We also see that, by comparison with 14(a), the $\operatorname{BR}\left(B_{s} \rightarrow \mu^{+} \mu^{-}\right)$constraint has moved to lower $A_{0}$, but the $m_{h}$ constraint has dropped even further, and $m_{h}>114 \mathrm{GeV}$ over the entire visible plane. The net result is a region compatible with all the constraints that extends from $A_{0} \sim-1850 \mathrm{GeV}$ down to $A_{0} \sim-2150 \mathrm{GeV}$ for $\mu \sim 400 \mathrm{GeV}$, with a coannihilation filament extending to larger $\mu$ when $A_{0} \sim-2200 \mathrm{GeV}$. Once again all of the WMAP strip in this panel is compatible with CDMS.

The larger allowed area of parameter space is reflected in panel (b) of Fig. 14, which has $\mu=400 \mathrm{GeV}$ and $A_{0}=-2000 \mathrm{GeV}$, as well as $\tan \beta=45$ and $M_{A}=$ $160 \mathrm{GeV}$ as before. In this case, we see that a substantial region of the WMAP strip with $m_{1 / 2} \sim 700 \mathrm{GeV}$ and a width $\delta m_{1 / 2} \sim 100 \mathrm{GeV}$, extending from $m_{0} \sim$ $750 \mathrm{GeV}$ to higher $m_{0}$ is allowed by all the other constraints. The $\operatorname{BR}\left(B_{s} \rightarrow \mu^{+} \mu^{-}\right)$and $m_{h}$ constraints have now moved to relatively low values of $m_{1 / 2}$, but 

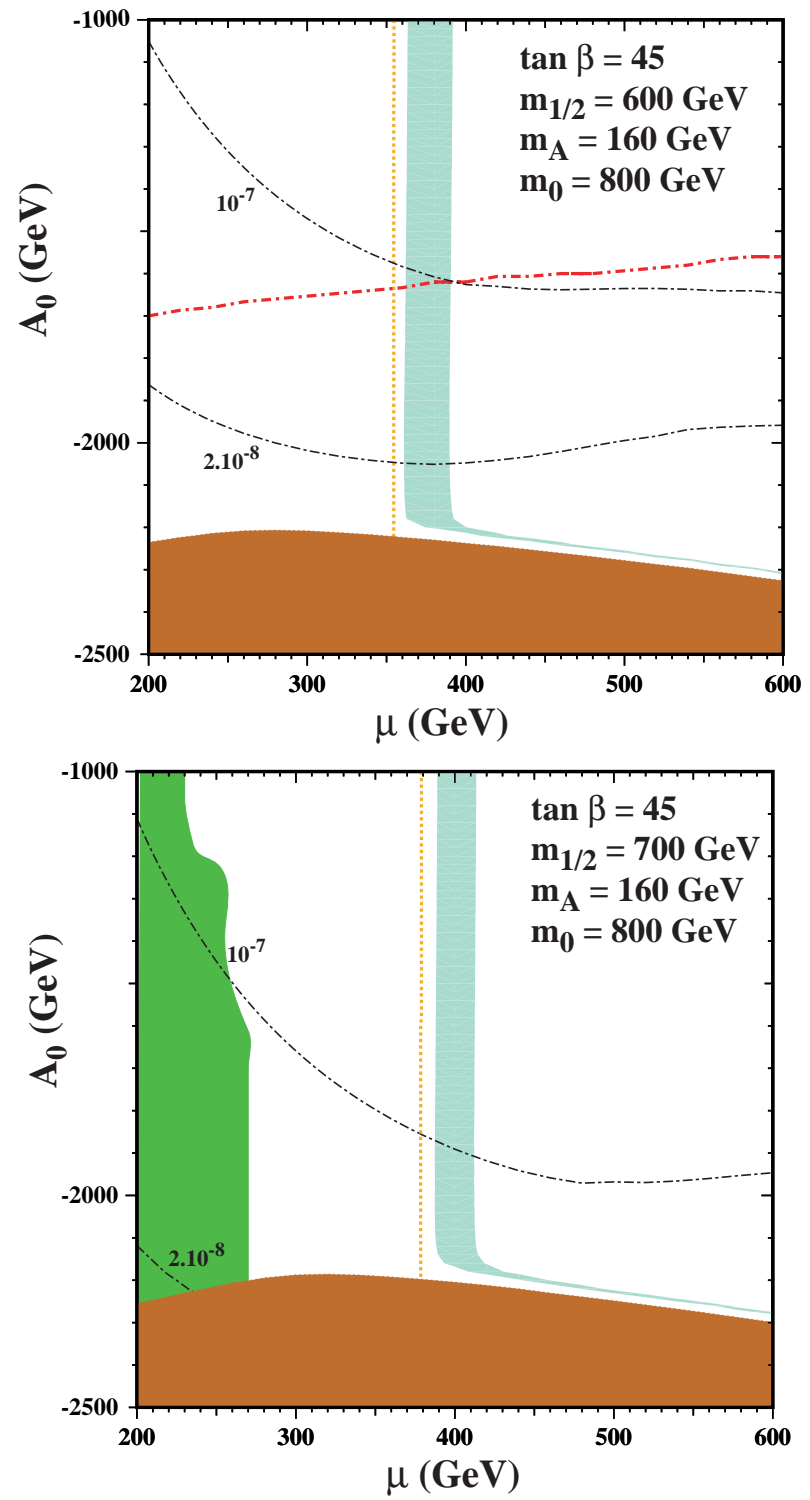

Fig. 15. The NUHM parameter space as a function of $\mu$ and $A_{0}$ for $m_{1 / 2}=600(700) \mathrm{GeV}$ and $m_{0}=800 \mathrm{GeV}$ in panels a (b). We fix $M_{A}=160 \mathrm{GeV}, \tan \beta=45, m_{t}=$ $171.4 \mathrm{GeV}$ and $m_{b}=4.25 \mathrm{GeV}$. For the description of the various lines and shaded areas, see the text.

we still find $\operatorname{BR}\left(B_{s} \rightarrow \mu^{+} \mu^{-}\right)>2 \times 10^{-8}$ and $m_{h}$ close to the LEP lower limit. The $(g-2)_{\mu}$ constraint truncates the allowed region at $m_{0} \sim 1050 \mathrm{GeV}$. Once again, the allowed region is compatible with CDMS (to the left of the (orange) dotted line) provided the strangeness contribution to the proton mass is small.

Clearly our anticipation for the discovery of supersymmetry or new physics is great. If the CDF hint of the Higgs boson is realized, we should expect to see numerous departures from the standard model including the decay of $B_{s}$ to $\mu^{+} \mu^{-}, \operatorname{BR}(b \rightarrow s \gamma)$ should show deviations from the standard model value, and dark matter should be discovered by CDMS or XENON10 in the near future. Similarly if an observation of new physics in any one of these areas occurs, we should be ready for flood of new data showing departures from the standard model.

\section{Acknowledgments}

I would like to thank J. Ellis, T. Hahn, S. Heinemeyer, P. Sandick, Y. Santoso, V. Spanos, A. Weber, and G. Weiglein for the many collaborations whose results were summarized here. This work was supported in part by DOE grant DE-FG02-94ER-40823.

\section{References}

1. S. M. Faber and J. J. Gallagher, Ann. Rev. Astron. Astrophys. 17 (1979) 135; A. Bosma, Ap. J. 86 (1981) 1825; V. C. Rubin, W. K. Ford and N. Thonnard, Ap. J. 238 (1980) 471; V. C. Rubin, D. Burstein, W. K. Ford and N. Thonnard, Ap. J. 289 (1985) 81; T. S. Van Albada and R. Sancisi, Phil. Trans. R. Soc. Land. A320 (1986) 447; M. Persic and P. Salucci, Ap. J. Supp. 99 (1995) 501; M. Persic, P. Salucci, and F. Stel, MNRAS 281 (1996) 27P.

2. D. N. Spergel et al. [WMAP Collaboration], Astrophys. J. Suppl. $\quad 170 \quad$ (2007) 377 arXiv:astro-ph/0603449.

3. A. G. Riess et al., A. J. 116 (1998) 1009; P. Garnovich et al. Ap. J. 509 (1998) 74; S. Perlmutter et al., Ap. J. 517 (1999) 565; J.L. Tonry et al. Ap. J. 594 (2003) 1; R.A. Knop et al. Ap. J. 598 (2003) 102; A. G. Riess et al. Ap. J. 607 (2004) 665.

4. D. J. Eisenstein et al. [SDSS Collaboration], Astrophys. J. 633 (2005) 560 arXiv:astro-ph/0501171.

5. D. Wittman, et al., Astrophys. J. 643, 128 (2006) arXiv:astro-ph/0507606.

6. D. Clowe, et al., Astrophys. J. 648 (2006) L109 arXiv:astro-ph/0608407.

7. K. A. Olive, arXiv:astro-ph/0301505

8. J. R. Ellis, S. Kelley and D. V. Nanopoulos, Phys. Lett. B 260 (1991) 131; U. Amaldi, W. de Boer and H. Furstenau, Phys. Lett. B 260 (1991) 447; P. Langacker and M. x. Luo, Phys. Rev. D 44 (1991) 817; C. Giunti, C. W. Kim and U. W. Lee, Mod. Phys. Lett. A 6 (1991) 1745.

9. For reviews, see: H. P. Nilles, Phys. Rep. 110 (1984) 1; A. Brignole, L. E. Ibanez and C. Munoz, arXiv:hep-ph/9707209, published in Perspectives on supersymmetry, ed. G. L. Kane, pp. 125-148.

10. M. Drees and M. M. Nojiri, Phys. Rev. D 47 (1993) 376 arXiv:hep-ph/9207234; H. Baer and M. Brhlik, Phys. Rev. D 53 (1996) 597 arXiv:hep-ph/9508321]; Phys. Rev. D 57 (1998) 567 arXiv:hep-ph/9706509]; H. Baer, M. Brhlik, M. A. Diaz, J. Ferrandis, P. Mercadante, P. Quintana and X. Tata, Phys. Rev. D 63 (2001) 015007 arXiv:hep-ph/0005027; A. B. Lahanas, D. V. Nanopoulos and V. C. Spanos, Mod. Phys. Lett. A 16 (2001) 1229 arXiv:hep-ph/0009065.

11. J. R. Ellis, T. Falk, K. A. Olive and M. Schmitt, Phys. Lett. B 388 (1996) 97 arXiv:hep-ph/9607292; Phys. Lett. B 413 (1997) 355 arXiv:hep-ph/9705444; J. R. Ellis, T. Falk, G. Ganis, K. A. Olive and M. Schmitt, Phys. Rev. D 58 (1998) 095002 
arXiv:hep-ph/9801445; V. D. Barger and C. Kao, Phys. Rev. D 57 (1998) 3131 arXiv:hep-ph/9704403; J. R. Ellis, T. Falk, G. Ganis and K. A. Olive, Phys. Rev. D 62 (2000) 075010 arXiv:hep-ph/0004169; V. D. Barger and C. Kao, Phys. Lett. B $\mathbf{5 1 8}$ (2001) 117 arXiv:hep-ph/0106189; L. Roszkowski, R. Ruiz de Austri and T. Nihei, JHEP 0108 (2001) 024 arXiv:hep-ph/0106334; A. B. Lahanas and V. C. Spanos, Eur. Phys. J. C 23 (2002) 185 arXiv:hep-ph/0106345; A. Djouadi, M. Drees and J. L. Kneur, JHEP 0108 (2001) 055 arXiv:hep-ph/0107316; U. Chattopadhyay, A. Corsetti and P. Nath, Phys. Rev. D 66 (2002) 035003 arXiv:hep-ph/0201001; J. R. Ellis, K. A. Olive and Y. Santoso, New Jour. Phys. 4 (2002) 32 arXiv:hep-ph/0202110; H. Baer, C. Balazs, A. Belyaev, J. K. Mizukoshi, X. Tata and Y. Wang, JHEP 0207 (2002) 050 arXiv:hep-ph/0205325; R. Arnowitt and B. Dutta, arXiv:hep-ph/0211417.

12. J. R. Ellis, T. Falk, G. Ganis, K. A. Olive and M. Srednicki, Phys. Lett. B 510 (2001) 236 arXiv:hep-ph/0102098.

13. J. R. Ellis, K. A. Olive, Y. Santoso and V. C. Spanos, Phys. Lett. B 565 (2003) 176 arXiv:hep-ph/0303043.

14. H. Baer and C. Balazs, arXiv:hep-ph/0303114 A. B. Lahanas and D. V. Nanopoulos, arXiv:hep-ph/0303130 U. Chattopadhyay, A. Corsetti and P. Nath, arXiv:hep-ph/0303201 C. Munoz, hep-ph/0309346

15. R. Barbieri, S. Ferrara and C.A. Savoy, Phys. Lett. 119B (1982) 343; For reviews, see: H. P. Nilles, Phys. Rep. 110 (1984) 1; A. Brignole, L. E. Ibanez and C. Munoz, arXiv:hep-ph/9707209, published in Perspectives on supersymmetry, ed. G. L. Kane, pp. 125148.

16. J. R. Ellis, K. A. Olive, Y. Santoso and V. C. Spanos, Phys. Lett. B 573 (2003) 162 arXiv:hep-ph/0305212; J. R. Ellis, K. A. Olive, Y. Santoso and V. C. Spanos, Phys. Rev. D 70 (2004) 055005 arXiv:hep-ph/0405110.

17. M. Olechowski and S. Pokorski, Phys. Lett. B 344, 201 (1995) arXiv:hep-ph/9407404; V. Berezinsky, A. Bottino, J. Ellis, N. Fornengo, G. Mignola and S. Scopel, Astropart. Phys. 5 (1996) 1, hep-ph/9508249 M. Drees, M. Nojiri, D. Roy and Y. Yamada, Phys. Rev. D 56 (1997) 276, [Erratumibid. D 64 (1997) 039901], hep-ph/9701219 M. Drees, Y. Kim, M. Nojiri, D. Toya, K. Hasuko and T. Kobayashi, Phys. Rev. D 63 (2001) 035008, hep-ph/0007202 P. Nath and R. Arnowitt, Phys. Rev. D 56 (1997) 2820, hep-ph/9701301, J. R. Ellis, T. Falk, G. Ganis, K. A. Olive and M. Schmitt, Phys. Rev. D 58 (1998) 095002 arXiv:hep-ph/9801445; J. R. Ellis, T. Falk, G. Ganis and K. A. Olive, Phys. Rev. D 62 (2000) 075010 arXiv:hep-ph/0004169; A. Bottino, F. Donato, N. Fornengo and S. Scopel, Phys. Rev. D 63 (2001) 125003, hep-ph/0010203 S. Profumo, Phys. Rev. D 68 (2003) 015006, hep-ph/0304071 D. Cerdeno and C. Munoz, JHEP 0410 (2004) 015, hep-ph/0405057, H. Baer, A. Mustafayev, S. Profumo, A. Belyaev and X. Tata, JHEP 0507 (2005) 065, hep-ph/0504001

18. J. Ellis, K. Olive and Y. Santoso, Phys. Lett. B 539, 107 (2002) arXiv:hep-ph/0204192; J. R. El- lis, T. Falk, K. A. Olive and Y. Santoso, Nucl. Phys. B 652, 259 (2003) arXiv:hep-ph/0210205.

19. J. Ellis, J.S. Hagelin, D.V. Nanopoulos, K.A. Olive and M. Srednicki, Nucl. Phys. B 238 (1984) 453; see also H. Goldberg, Phys. Rev. Lett. 50 (1983) 1419.

20. J. Ellis, T. Falk, and K.A. Olive, Phys. Lett. B444 (1998) 367 arXiv:hep-ph/9810360; J. Ellis, T. Falk, K.A. Olive, and M. Srednicki, Astr. Part. Phys. 13 (2000) 181 [Erratum-ibid. 15 (2001) 413] arXiv:hep-ph/9905481.

21. Joint LEP 2 Supersymmetry Working Group, Combined LEP Chargino Results, up to $208 \mathrm{GeV}$, http://lepsusy.web.cern.ch/lepsusy/www/inos_ moriond01/charginos_pub.html.

22. Joint LEP 2 Supersymmetry Working Group, Combined LEP Selectron/Smuon/Stau Results, 183-208 GeV, http://lepsusy.web.cern.ch/lepsusy/www/sleptons_ summer02/slep_2002.html.

23. LEP Higgs Working Group for Higgs boson searches, OPAL Collaboration, ALEPH Collaboration, DELPHI Collaboration and L3 Collaboration, Phys. Lett. B 565 (2003) 61 arXiv:hep-ex/0306033. Search for neutral Higgs bosons at LEP, paper submitted to ICHEP04, Beijing, LHWG-NOTE-200401, ALEPH-2004-008, DELPHI-2004-042, L3-NOTE2820, OPAL-TN-744, http://lephiggs . web.cern.ch/LEPHIGGS/papers/ August2004_MSSM/index.html.

24. S. Heinemeyer, W. Hollik and G. Weiglein, Comput. Phys. Commun. 124 (2000) 76 arXiv:hep-ph/9812320; S. Heinemeyer, W. Hollik and G. Weiglein, Eur. Phys. J. C 9 (1999) 343 arXiv:hep-ph/9812472.

25. S. Chen et al. [CLEO Collaboration], Phys. Rev. Lett. 87 (2001) 251807 arXiv:hep-ex/0108032; P. Koppenburg et al. [Belle Collaboration], Phys. Rev. Lett. 93 (2004) 061803 arXiv:hep-ex/0403004. B. Aubert et al. [BaBar Collaboration], arXiv:hep-ex/0207076, E. Barberio et al. [Heavy Flavor Averaging Group (HFAG)], arXiv:hep-ex/0603003.

26. C. Degrassi, P. Gambino and G. F. Giudice, JHEP 0012 (2000) 009 arXiv:hep-ph/0009337, as implemented by P. Gambino and G. Ganis.

27. [The Muon g-2 Collaboration], Phys. Rev. Lett. 92 (2004) 161802, hep-ex/0401008 G. Bennett et al. [The Muon g-2 Collaboration], Phys. Rev. D $\mathbf{7 3}$ (2006) 072003 arXiv:hep-ex/0602035.

28. M. Davier, S. Eidelman, A. Höcker and Z. Zhang, Eur. Phys. J. C 31 (2003) 503, hep-ph/0308213, see also M. Knecht, hep-ph/0307239 K. Melnikov and A. Vainshtein, Phys. Rev. D70 (2004) 113006 arXiv:hep-ph/0312226. J. de Troconiz and F. Yndurain, hep-ph/0402285. M. Passera, arXiv:hep-ph/0411168, K. Hagiwara, A. Martin, D. Nomura and T. Teubner, Phys. Lett. B 649 (2007) 173 arXiv:hep-ph/0611102; M. Davier, arXiv:hep-ph/0701163 F. Jegerlehner, arXiv:hep-ph/0703125 J. Miller, E. de Rafael and B. Roberts, Rept. Prog. Phys. 70 (2007) 795 arXiv:hep-ph/0703049; S. Eidelman, talk given at the ICHEP06, Moscow, July 2006, see: http://ichep06.jinr.ru/reports/333_6s1_9p30_ Eidelman.pdf 
29. J. L. Feng, K. T. Matchev and T. Moroi, Phys. Rev. D 61 (2000) 075005 arXiv:hep-ph/9909334.

30. J. Ellis, A. Ferstl and K. A. Olive, Phys. Lett. B 481, 304 (2000) arXiv:hep-ph/0001005;

31. M. A. Shifman, A. I. Vainshtein and V. I. Zakharov, Phys. Lett. 78B, 443 (1978);

A. I. Vainshtein, V. I. Zakharov and M. A. Shifman, Usp. Fiz. Nauk 130, 537 (1980).

32. J. Gasser, H. Leutwyler, and M. E. Sainio, Phys. Lett. B253, 252 (1991); M. Knecht, hep-ph/9912443 M. E. Sainio, PiN Newslett. 16, 138 (2002) arXiv:hep-ph/0110413.

33. J. Ellis, S. Heinemeyer, K. Olive and G. Weiglein, JHEP 0502013 (2005) arXiv:hep-ph/0411216.

34. J. R. Ellis, S. Heinemeyer, K. A. Olive and G. Weiglein, JHEP 0605, 005 (2006) arXiv:hep-ph/0602220.

35. J. Ellis, S. Heinemeyer, K. Olive, A.M. Weber and G. Weiglein, JHEP 0708 (2007) 083 arXiv:0706.0652 [hep-ph]].

36. S. Heinemeyer, these proceedings.

37. G. Weiglein, these proceedings.

38. J. Ellis, K. A. Olive, Y. Santoso and V. C. Spanos, arXiv:hep-ph/0502001.

39. D. Akerib et al. [CDMS Collaboration], Phys. Rev. Lett. 96 (2006) 011302, astro-ph/0509259.

40. J. Angle et al. [XENON Collaboration], arXiv:0706.0039 [astro-ph], see also: http://xenon.astro.columbia.edu/ .

41. K. Choi, K. S. Jeong and K. i. Okumura, JHEP 0509 (2005) 039 arXiv:hep-ph/0504037; M. Endo, M. Yamaguchi and K. Yoshioka, Phys. Rev. D 72 (2005) 015004 arXiv:hep-ph/0504036; A. Falkowski, O. Lebedev and Y. Mambrini, JHEP 0511 (2005) 034 arXiv:hep-ph/0507110; R. Kitano and Y. Nomura, Phys. Lett. B 631 (2005) 58 arXiv:hep-ph/0509039; R. Kitano and Y. Nomura, Phys. Rev. D 73 (2006) 095004 arXiv:hep-ph/0602096; A. Pierce and J. Thaler, JHEP 0609 (2006) 017 arXiv:hep-ph/0604192; K. Kawagoe and M. M. Nojiri, arXiv:hep-ph/0606104]; H. Baer, E.-K. Park, X. Tata and T. T. Wang, JHEP 0608 (2006) $041 \quad$ arXiv:hep-ph/0604253; K. Choi, K. Y. Lee, Y. Shimizu, Y. G. Kim and K. i. Okumura, JCAP 0612 (2006) 017 arXiv:hep-ph/0609132; O. Lebedev, V. Lowen, Y. Mambrini, H. P. Nilles and M. Ratz, JHEP 0702 (2007) 063 arXiv:hep-ph/0612035.

42. H. Itoh, N. Okada and T. Yamashita, arXiv:hep-ph/0606156.

43. S. Kachru, R. Kallosh, A. Linde and S. P. Trivedi, Phys. Rev. D 68 (2003) 046005 arXiv:hep-th/0301240; K. Choi, A. Falkowski, H. P. Nilles and M. Olechowski, Nucl. Phys. B 718 (2005) 113 arXiv:hep-th/0503216.

44. J. R. Ellis, K. A. Olive and P. Sandick, Phys. Lett. B 642 (2006) 389 arXiv:hep-ph/0607002 ; JHEP 0706 (2007) 079 arXiv:0704.3446 [hep-ph]].

45. P. Sandick, these proceedings.

46. J. Polonyi, Budapest preprint KFKI-1977-93 (1977).

47. J. R. Ellis, K. A. Olive, Y. Santoso and V. C. Spanos, Phys. Lett. B 588 (2004) 7 arXiv:hep-ph/0312262; J. L. Feng, A. Rajaraman and F. Takayama, Phys. Rev. Lett. 91 (2003) 011302 arXiv:hep-ph/0302215; J. L. Feng, S. Su and F. Takayama, Phys. Rev. D 70 (2004) 075019 arXiv:hep-ph/0404231.
48. R. H. Cyburt, J. R. Ellis, B. D. Fields and K. A. Olive, Phys. Rev. D 67 (2003) 103521 arXiv:astro-ph/0211258; J. L. Feng, S. f. Su and F. Takayama, Phys. Rev. D 70 (2004) 063514 arXiv:hep-ph/0404198; J. R. Ellis, K. A. Olive and E. Vangioni, Phys. Lett. B 619, 30 (2005) arXiv:astro-ph/0503023; K. Kohri, T. Moroi and A. Yotsuyanagi, Phys. Rev. D 73 (2006) 123511 arXiv:hep-ph/0507245; D. G. Cerdeno, K. Y. Choi, K. Jedamzik, L. Roszkowski and R. Ruiz de Austri, JCAP $0606 \quad$ (2006) 005 arXiv:hep-ph/0509275; F. D. Steffen, JCAP 0609, 001 (2006) arXiv:hep-ph/0605306.

49. M. Pospelov, Phys. Rev. Lett. 98 (2007) 231301 arXiv:hep-ph/0605215; K. Hamaguchi, T. Hatsuda, M. Kamimura, Y. Kino and T. T. Yanagida, Phys. Lett. B 650 (2007) 268 arXiv:hep-ph/0702274; C. Bird, K. Koopmans and M. Pospelov, arXiv:hep-ph/0703096.

50. R. H. Cyburt, J. R. Ellis, B. D. Fields, K. A. Olive and V. C. Spanos, JCAP $0611 \quad$ (2006) 014 arXiv:astro-ph/0608562.

51. K. Jedamzik, Phys. Rev. D 70 (2004) 063524 arXiv:astro-ph/0402344; K. Jedamzik, Phys. Rev. D 70 (2004) 083510 arXiv:astro-ph/0405583.

52. G. Sigl, K. Jedamzik, D. N. Schramm and V. S. Berezinsky, Phys. Rev. D 52 (1995) 6682 arXiv:astro-ph/9503094.

53. J. Ellis, T. Hahn, S. Heinemeyer, K. A. Olive and G. Weiglein, arXiv:0709.0098 [hep-ph].

54. A. Abulencia et al. [CDF Collaboration], Phys. Rev. Lett. 96 (2006) 011802, hep-ex/0508051.

55. V. Abazov et al. [D0 Collaboration], Phys. Rev. Lett. 97 (2006) 121802, hep-ex/0605009

56. A. Abulencia et al. [CDF Collaboration], Phys. Rev. Lett. 96 (2006) 042003, hep-ex/0510065.

57. CDF Collaboration, CDF note 8676 , see: http://www-cdf.fnal.gov/ aa/mssm_htt_1fb/note/ cdf8676.pdf.

58. D0 Collaboration, D0 Note 5331-CONF, see: http://www-d0.fnal.gov/cgi-bin/d0note?5331

59. M. Carena, S. Heinemeyer, C. Wagner and G. Weiglein, Eur. Phys. J. C 45 (2006) 797, hep-ph/0511023.

60. A. Djouadi and Y. Mambrini, JHEP 0612 (2006) 001, hep-ph/0609234.

61. J. R. Ellis, S. Heinemeyer, K. A. Olive and G. Weiglein, arXiv:0706.0977 [hep-ph].

62. D. Acosta et al. [CDF Collaboration], Phys. Rev. Lett. 93 (2004) 032001 arXiv:hep-ex/0403032; V. M. Abazov et al. [D0 Collaboration], Phys. Rev. Lett. 94, 071802 (2005) arXiv:hep-ex/0410039; The D0 Collaboration, D0note, 4733-CONF; http://www-d0.fnal.gov/Run2Physics/WWW/results /prelim/B/B21/B21.pdf; M. Herndon, The CDF and D0 Collaborations, FERMILAB-CONF04-391-E. Published Proceedings 32nd International Conference on High-Energy Physics (ICHEP 04), Beijing, China, August 16-22, 2004; The CDF Collaboration, CDF note 7670; http://www-cdf.fnal.gov/physics/new/bottom /050407.blessed-bsmumu/.

63. J. R. Ellis, K. A. Olive and V. C. Spanos, Phys. Lett. B 624 (2005) 47 arXiv:hep-ph/0504196; J. R. Ellis, K. A. Olive, Y. Santoso and V. C. Spanos, JHEP 0605, 063 (2006) arXiv:hep-ph/0603136. 